Supporting Information

\title{
Sub-10 fs Intervalley Exciton Coupling in Monolayer MoS2 Revealed by Helicity-Resolved Two-Dimensional Electronic Spectroscopy
}

Lawson T. Lloyd, ${ }^{1,2,3}$ Ryan E. Wood, ${ }^{1,2,3}$ Fauzia Mujid, ${ }^{1,2}$ Siddhartha Sohoni, ${ }^{1,2,3}$ Karen L. Ji, ${ }^{1,2,3}$ Po-Chieh Ting, ,2,3 Jacob S. Higgins, ${ }^{1,2,3}$ Jiwoong Park, ${ }^{1,2,4}$ and Gregory S. Engel ${ }^{1,2,3, *}$

${ }^{1}$ Department of Chemistry, The University of Chicago, Chicago, Illinois 60637, USA

${ }^{2}$ James Franck Institute, The University of Chicago, Chicago, Illinois 60637, USA

${ }^{3}$ Institute for Biophysical Dynamics, The University of Chicago, Chicago, Illinois 60637, USA

${ }^{4}$ Pritzker School of Molecular Engineering, The University of Chicago, Chicago, Illinois 60637, USA

*Corresponding author: gsengel@uchicago.edu

1. Material characterization

2. Femtosecond pulse characterization

3. Additional 2DES spectra

4. Additional 2DES waiting time traces

5. 2DES measurements on strain-relieved sample growths

6. Estimating the carrier-density in 2DES measurements

7. Coupling features and cross-peaks in two-dimensional spectroscopy 


\section{Material characterization}

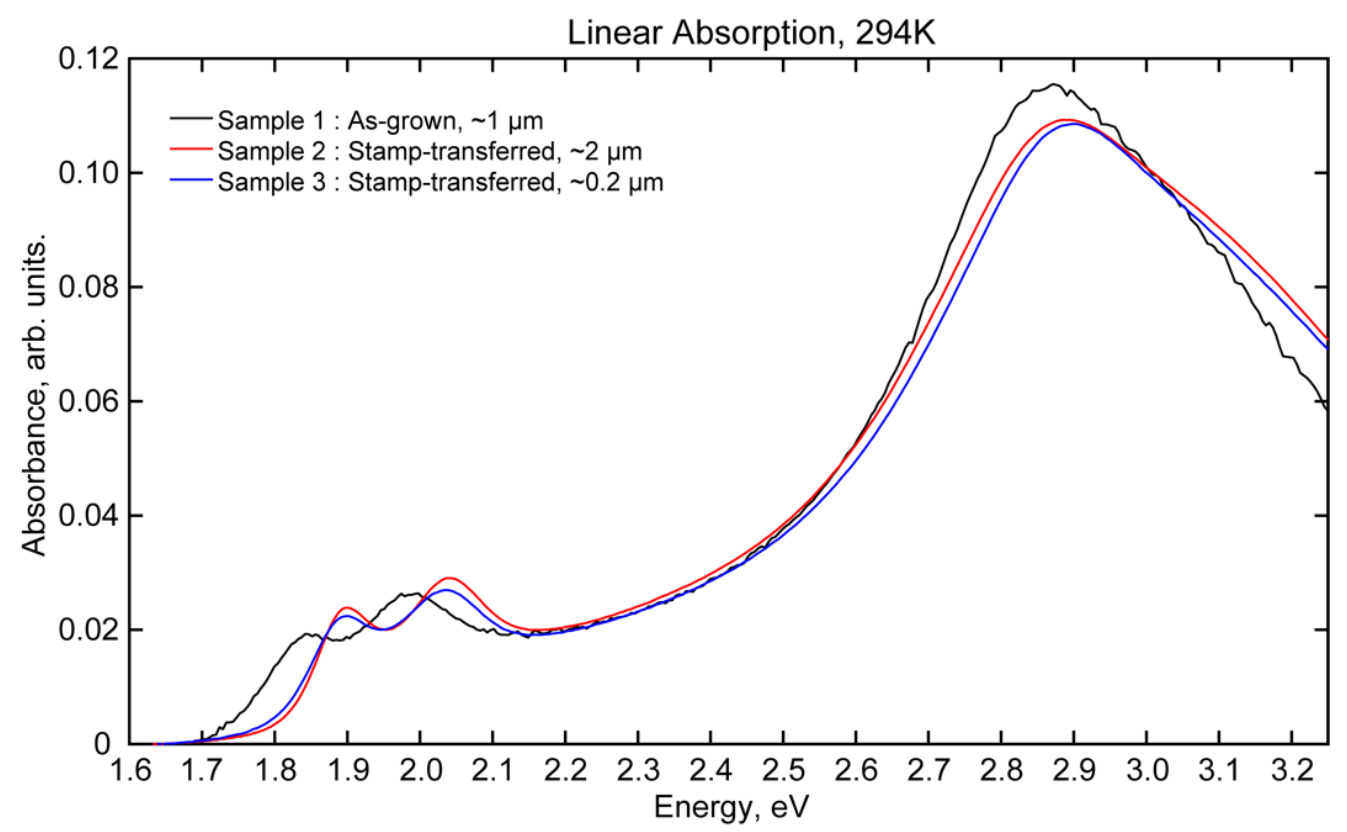

Figure S1. Linear absorption spectrum of MOCVD-grown monolayer $\mathrm{MoS}_{2}$ samples at $294 \mathrm{~K}$ after scatter baseline subtraction (Agilent Cary 5000). The spectral shifts after stamp-transfer are the result of relieving inherent strain acquired during the growth process.

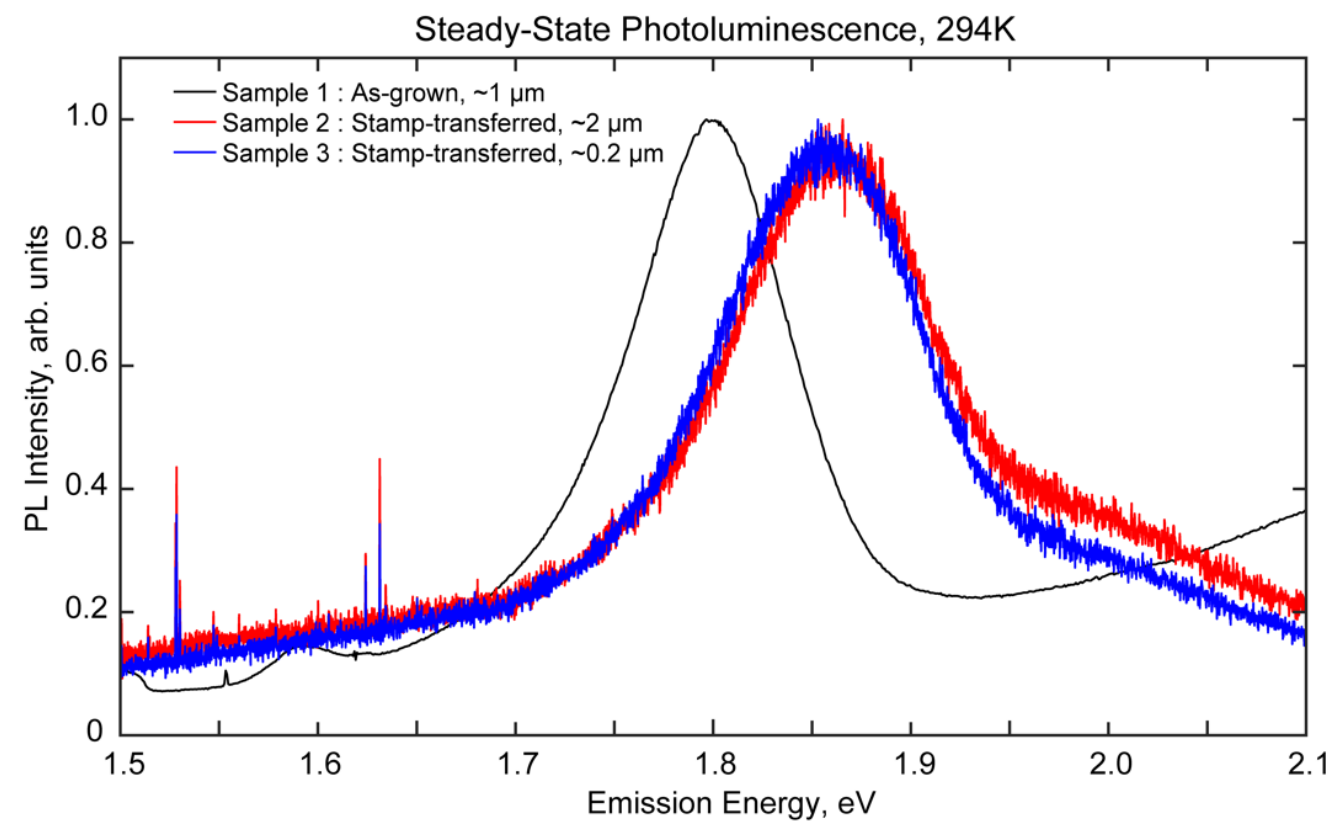

Figure S2. Photoluminescence (PL) spectra of the monolayer $\mathrm{MoS}_{2}$ samples at 294K. The PL spectra of Sample 2 and Sample 3 were acquired with a confocal Raman microscope (HORIBA LabRAM HR Evolution, excitation wavelength of $532 \mathrm{~nm}$ ) and the Sample 1 spectrum was acquired with a widefield spectrofluorometer (HORIBA Fluorolog-3, excitation wavelength of $520 \mathrm{~nm}$ ). 


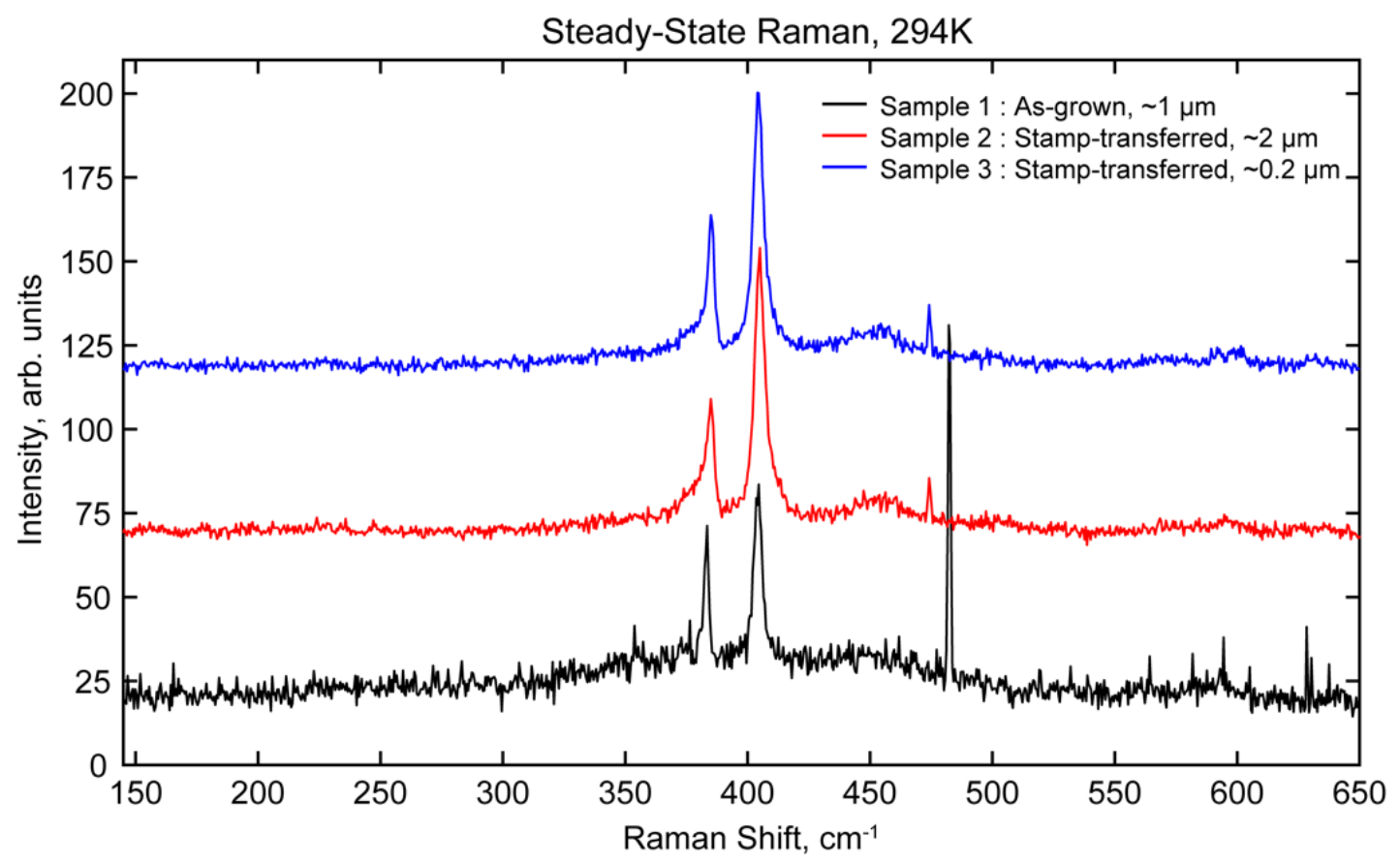

Figure S3. Raman spectra of monolayer $\mathrm{MoS}_{2}$ samples at 294K (HORIBA LabRAM HR Evolution, excitation wavelength of $532 \mathrm{~nm}$ ). The data are offset by $50 \mathrm{arb}$. units for clarity.

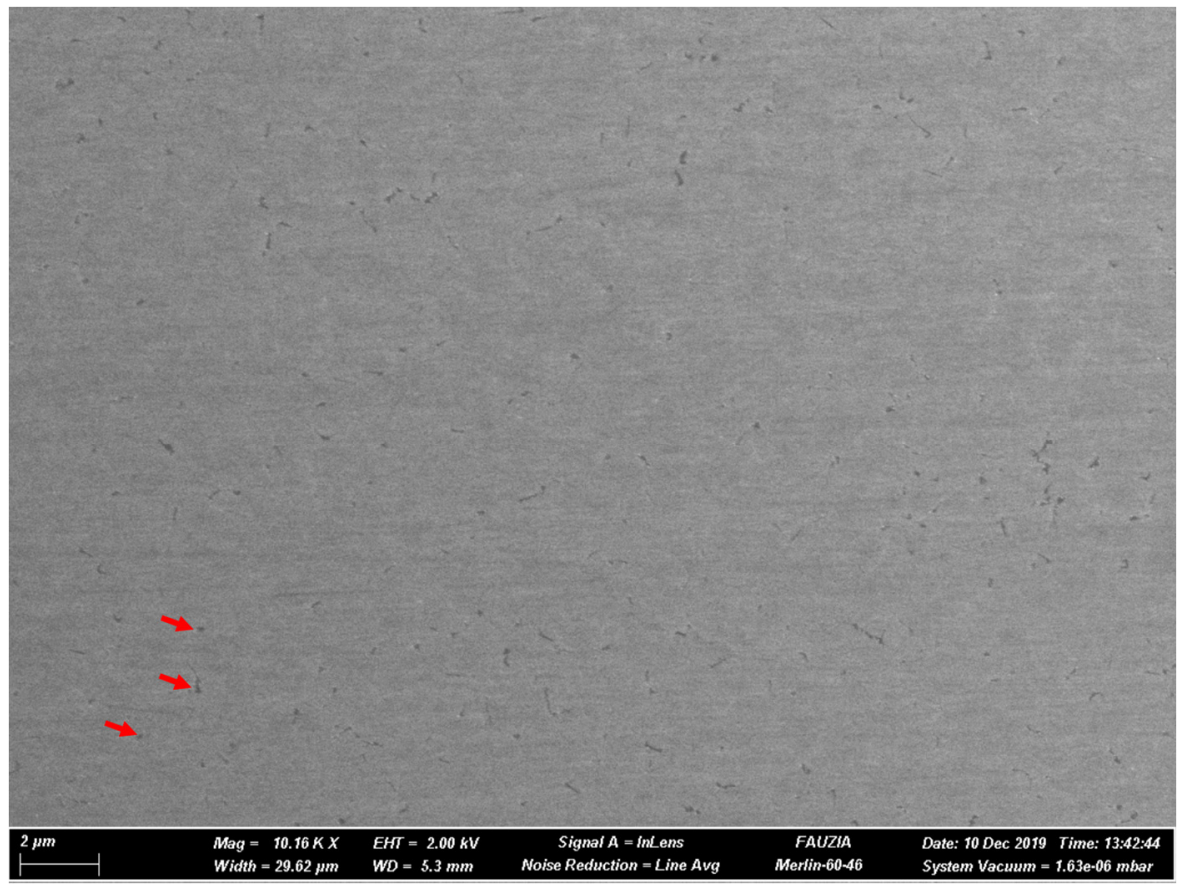

Figure S4. SEM image of a $\mathrm{MoS}_{2}$ film similar to the ones used in this study. The image is taken from a film that was grown slightly longer than the ideal growth time for a single $\mathrm{MoS}_{2}$ layer, since the pure monolayer $\mathrm{MoS}_{2}$ surface is flat and featureless. The darker spots in the image (e.g. those indicated by the red arrows) are islands of bilayer of $\mathrm{MoS}_{2}$ growing on top of the uniform monolayer $\mathrm{MoS}_{2}$ background. 


\section{Femtosecond pulse characterization}

(a)

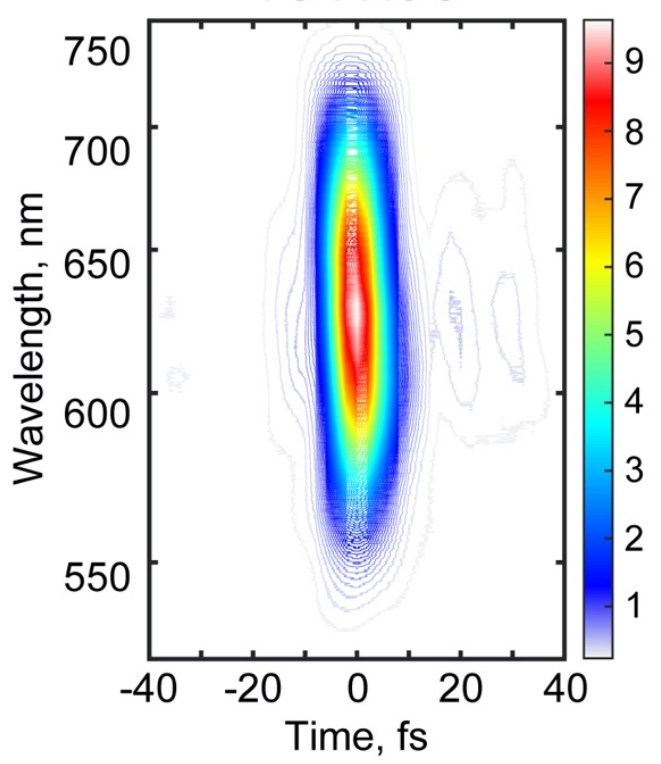

(b) Integrated TG-FROG

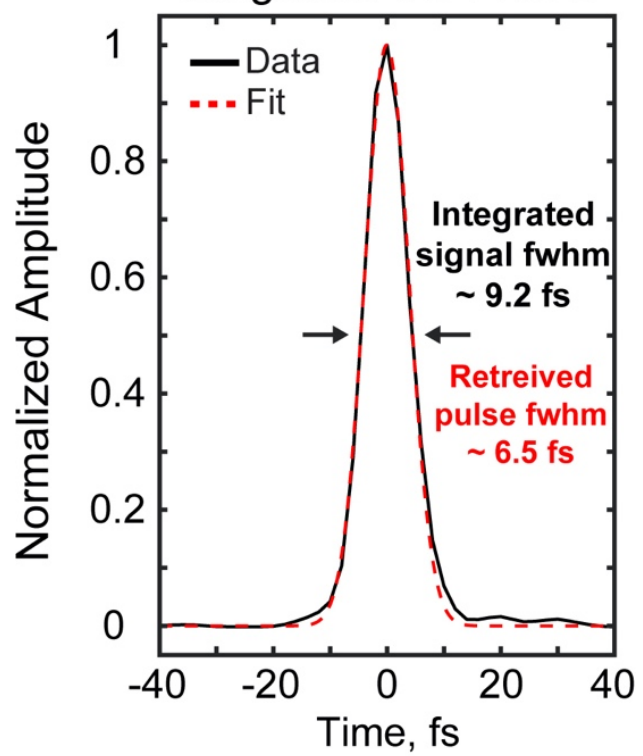

Figure S5. Representative femtosecond pulse characterization. (a) TG-FROG measurement in a glass slide at the sample position in the 'BOXCARS' 2DES experimental geometry. (b) Integrating over the wavelength dimension and fitting a Gaussian function to the time trace estimates a $\sim 9.2 \mathrm{fs}$ full-width at half-maximum (fwhm) of the integrated signal, corresponding to an estimated pulse fwhm of $\sim 6.5 \mathrm{fs}$.

(a)

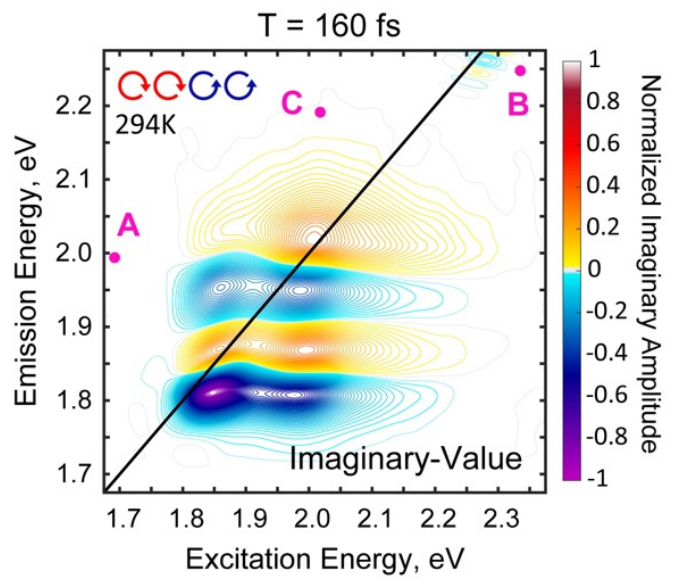

(b)
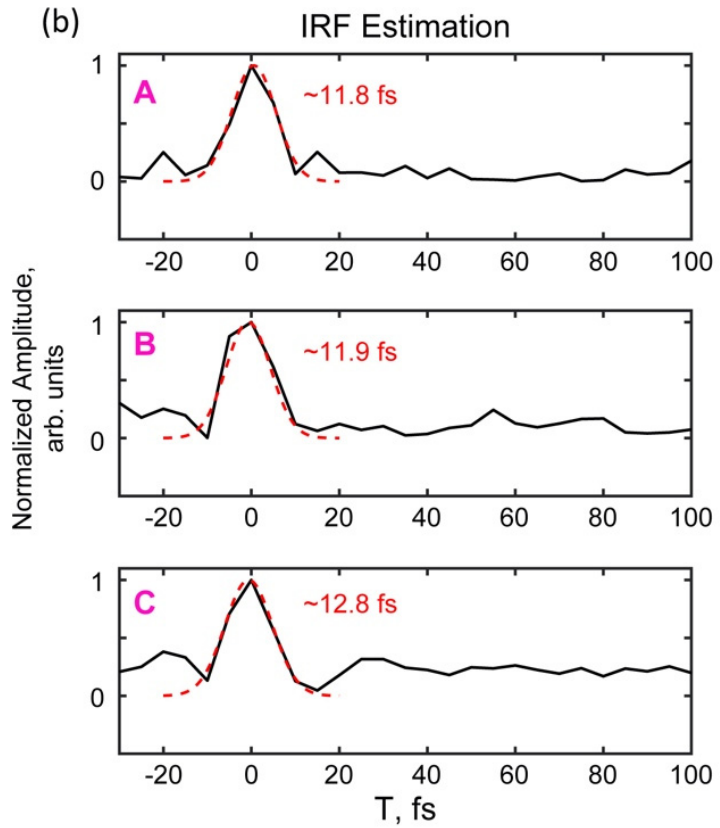

Figure S6. Estimating the instrument response function (IRF) using the 2DES data. (a) Imaginary-valued 2DES spectrum, which contains dispersive signal contributions. (b) Time traces from the points A-C labelled in the 2D spectrum show an estimated $\sim 12$ fs IRF from the imaginary-valued data. 


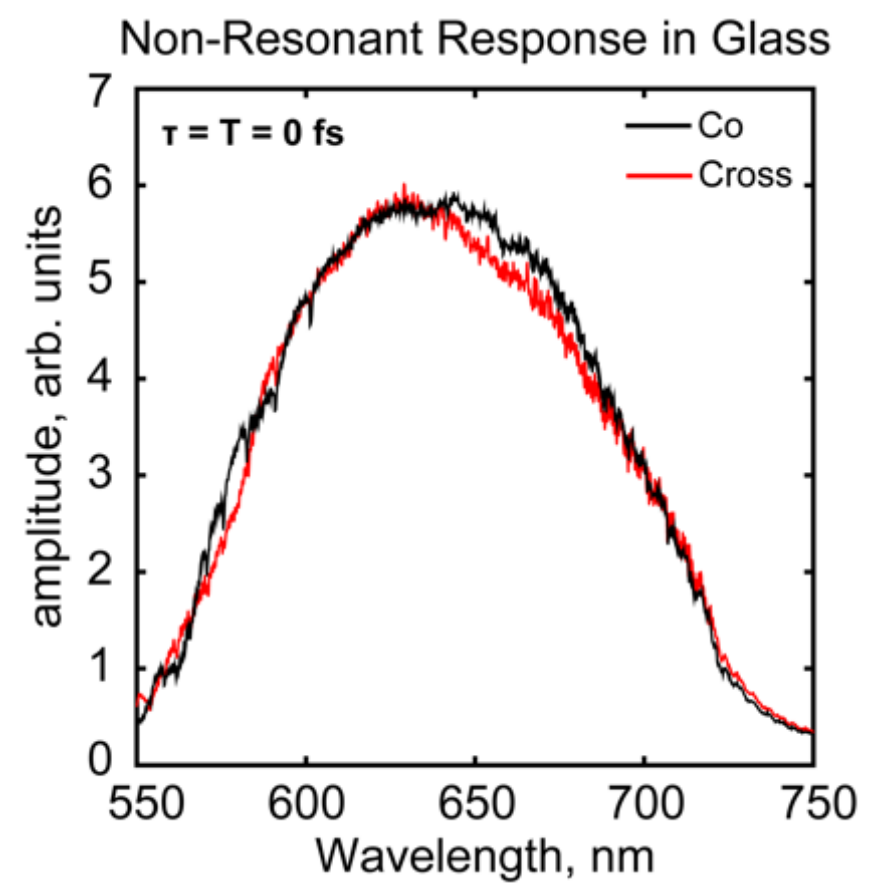

Figure S7. Control experiments of co- and cross-circularly polarized pulse sequences, labelled as 'Co' and 'Cross', respectively. Comparison of the nonresonant response in a glass slide at zero time delay shows negligible difference in the intensity or spectral shape between the two polarization sequences.

(a)

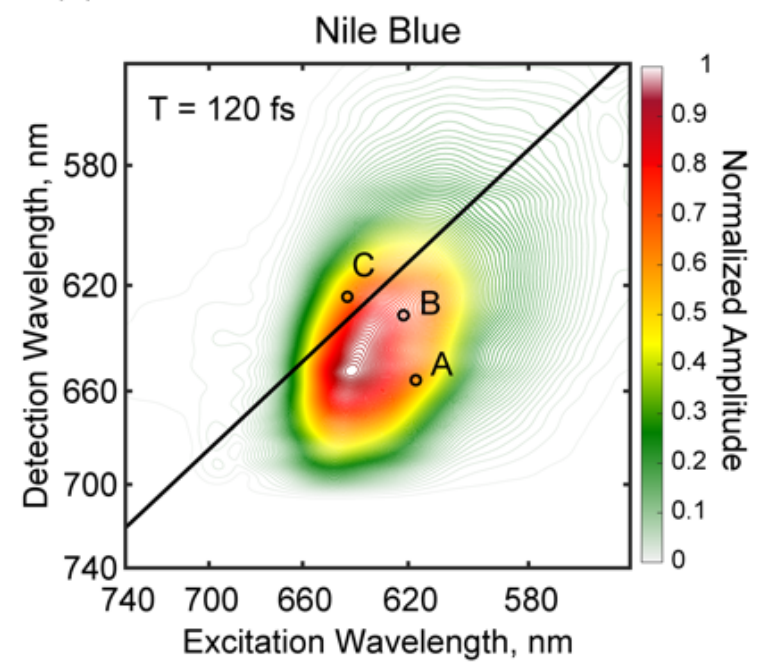

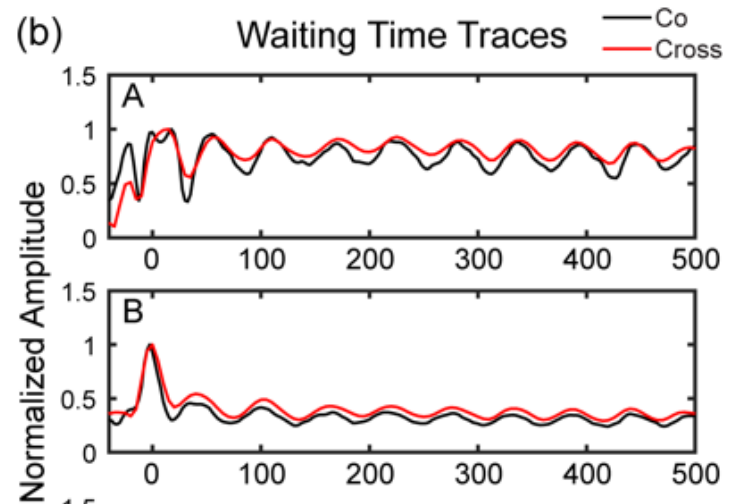

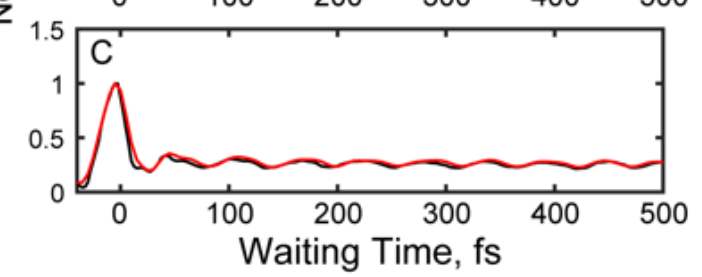

Figure S8. Control experiments of co- and cross-circularly polarized pulse sequences, labelled as 'Co' and 'Cross', respectively. ( $(a, b)$ Co- and cross-circularly polarized 2DES measurements of the laser dye Nile Blue show no appreciable differences in either the absolute intensity near $\mathrm{T}=0 \mathrm{fs}$ or in the subsequent waiting time dynamics. Oscillatory dynamics arise from vibrational wavepackets. Absolute-value spectra are shown. 
Polarization sequences in the 2DES experiments were controlled using a broadband halfwave plate in each beam path as well as a common broadband quarter-wave plate for all beams directly before the sample position. Setting the half-wave plates to all-collinear polarizations as verified by a Glan-Thompson and power meter resulted in the co-circularly polarized pulse sequence (i.e. $\sigma_{+} \sigma_{+} \sigma_{+} \sigma_{+}$) at the sample position after inserting the common-path quarter-wave plate, whereas setting the linear polarizations of beams 1 and 2 perpendicular to beam 3 and the LO resulted in the cross-circularly polarized pulse sequence (i.e. $\left.\sigma_{+} \sigma_{+} \sigma_{-} \sigma_{-}\right)$at the sample position.

To measure the purity of the circular polarization for a given beam, the maximum and minimum powers measured on a power meter were recorded after a full rotation of the GlanThompson polarizer placed at the sample position. All beams used in the experiment had circular polarization purity $>90 \%$ defined as the ratio of the minimum and maximum powers recorded during rotation of the Glan-Thompson. Furthermore, analysis of the laser spectrum of a circularly polarized excitation beam shows small $(<5 \%)$ deviation as a function of the Glan-Thompson angle (Figure S9).

(a)

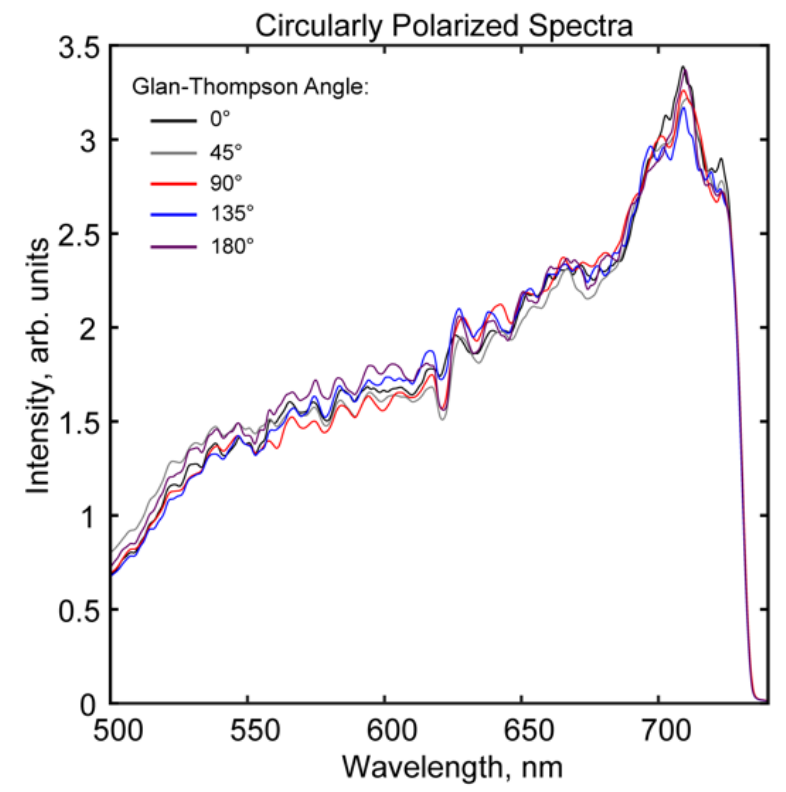

(b)

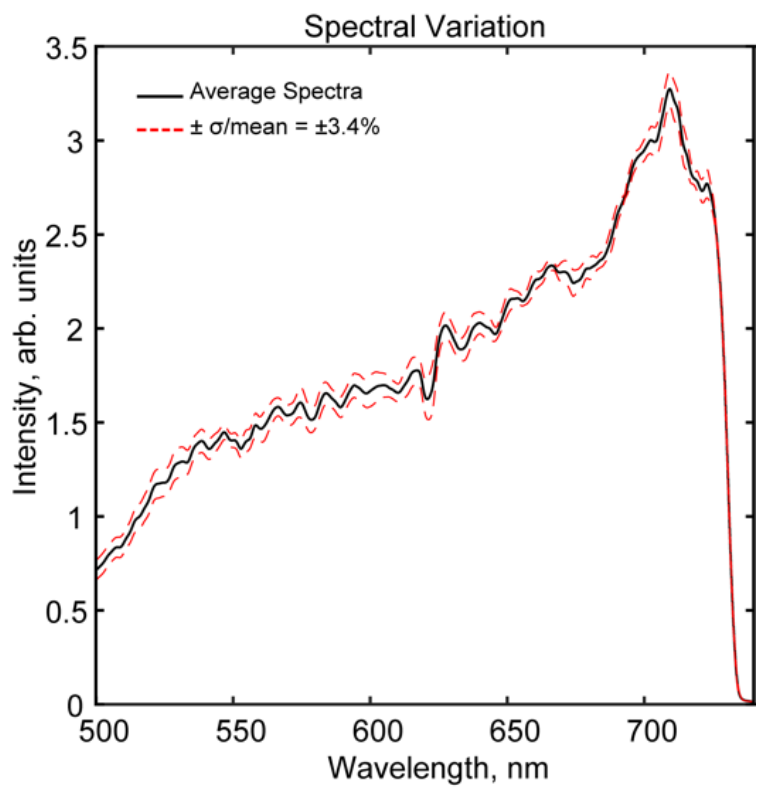

Figure S9. Representative circularly polarized characterization of a broadband laser pulse. (a) Laser spectra of a circularly excitation beam as a function of Glan-Thompson angle placed before the spectrometer and after the waveplates. (b) The spectra show an average of $\sim 4 \%$ deviation, consistent with typical $\sim 90 \%$ purity values measured using a Glan-Thompson and power meter combination when performing the alignment. Importantly, the data demonstrate negligible spectral variation as a function of Glan-Thompson polarizer angle when characterizing circularly polarized laser beams. 


\section{Additional 2DES spectra}
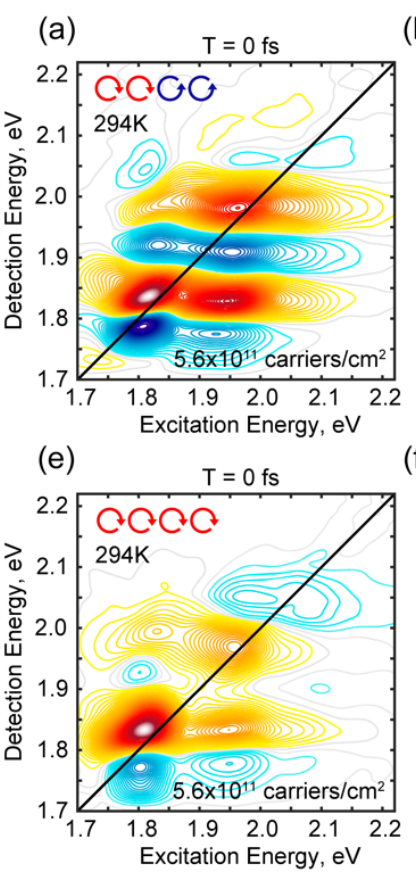

(b)

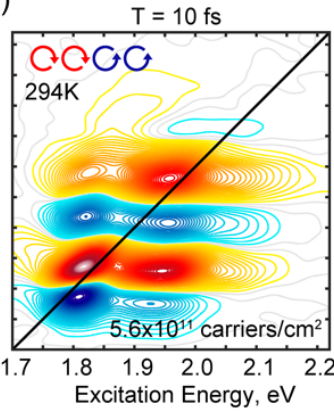

(f)

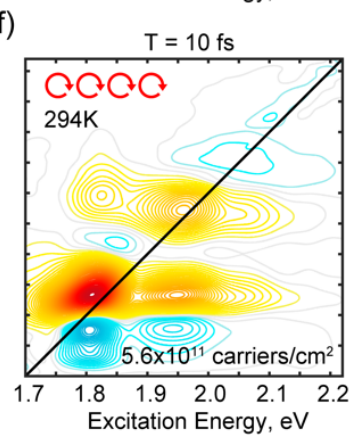

(c)

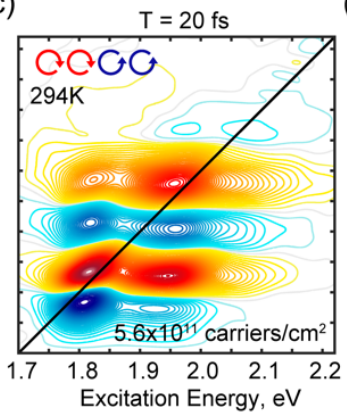

(g)

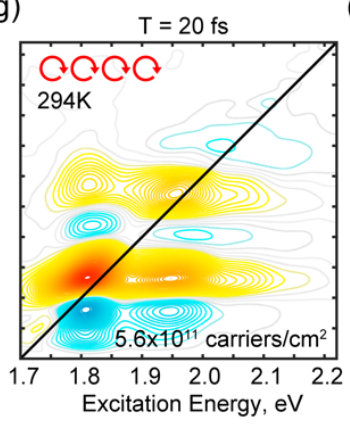

(d)

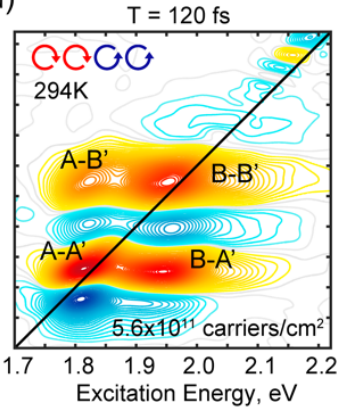

(h)

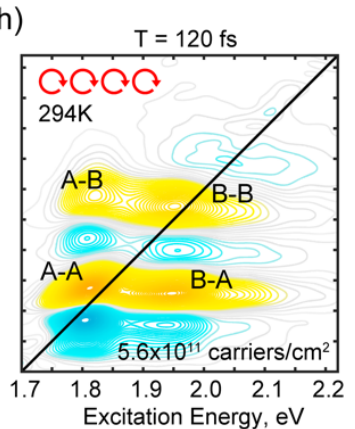

Figure S10. Absorptive 2DES maps for cross- (a-d) and co-polarized (e-h) room-temperature experiments at an estimated carrier density of $5.6 \times 10^{11}$ carriers $/ \mathrm{cm}^{2}$ for waiting time delays $\mathrm{T}=0,10,20$, and $120 \mathrm{fs}$. The spectra are normalized to the global maximum of the dataset for each experiment. The displayed data is an average of $\mathrm{n}=6$ and $\mathrm{n}=5$ datasets for the cross- and co-polarized experiments, respectively.
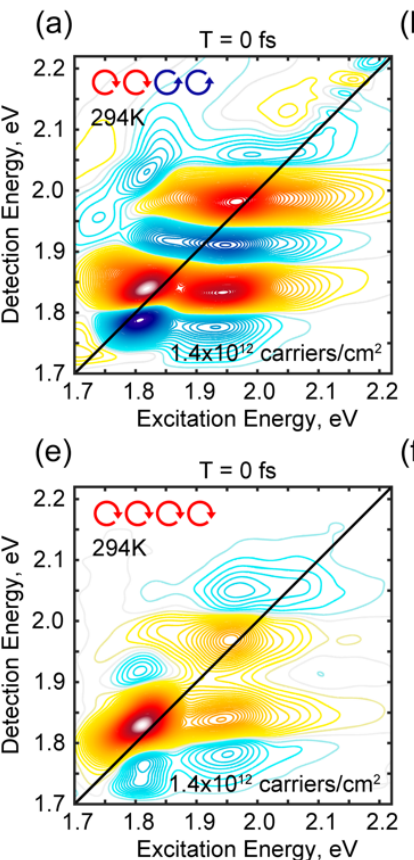

(b)

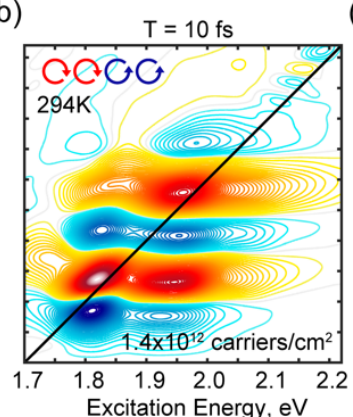

(f)

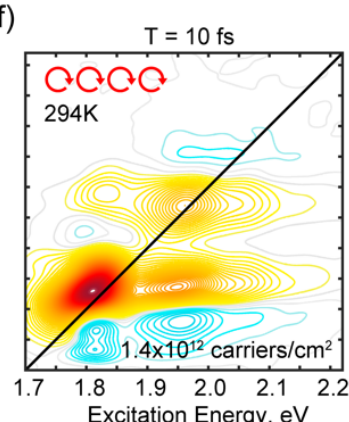

(c)

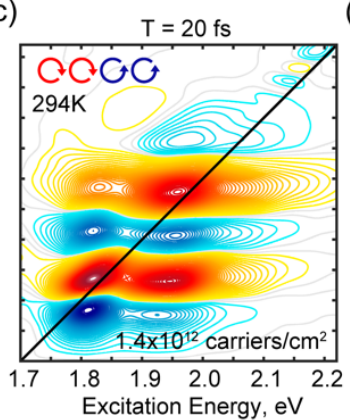

(g)

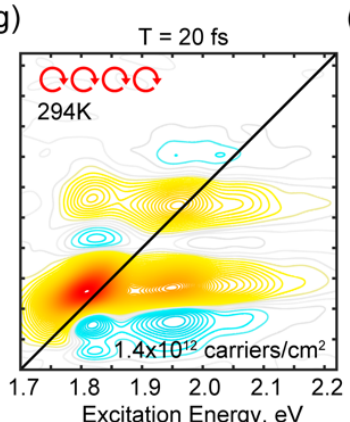

(d)

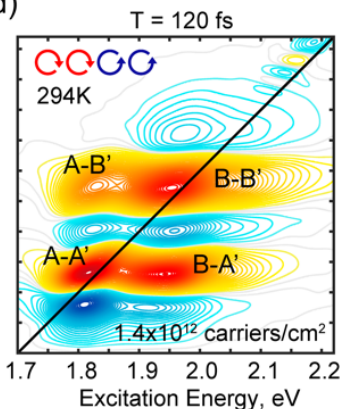

(h)

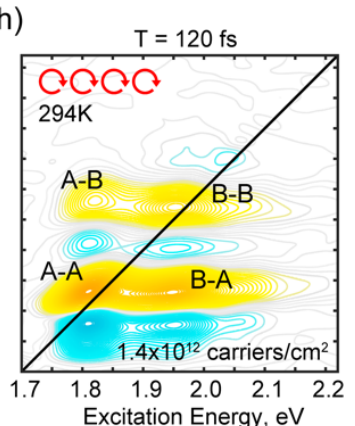

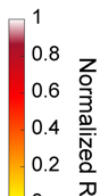

Figure S11. Absorptive 2DES maps for cross- (a-d) and co-polarized (e-h) room-temperature experiments at an estimated carrier density of $1.4 \times 10^{12}$ carriers $/ \mathrm{cm}^{2}$ for waiting time delays $\mathrm{T}=0,10,20$, and $120 \mathrm{fs}$. The spectra are normalized to the global maximum of the dataset for each experiment. The displayed data is an average of $\mathrm{n}=8$ and $\mathrm{n}=3$ datasets for the cross- and co-polarized experiments, respectively. 

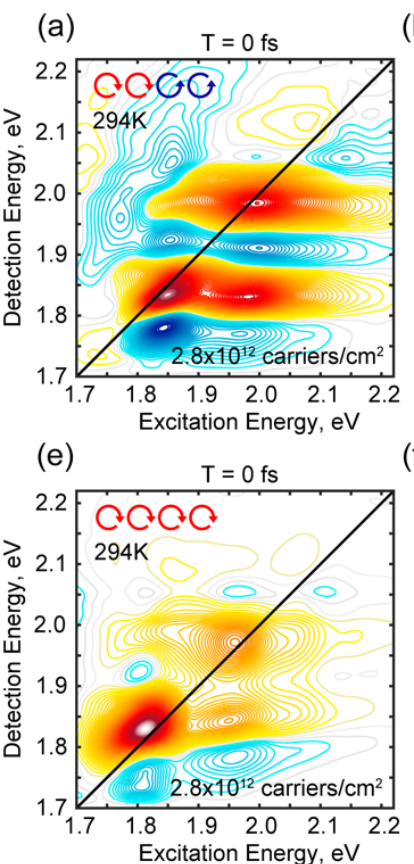

(b)

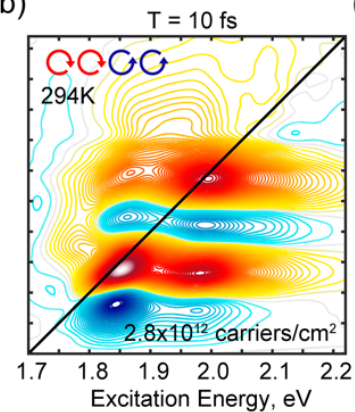

(f)

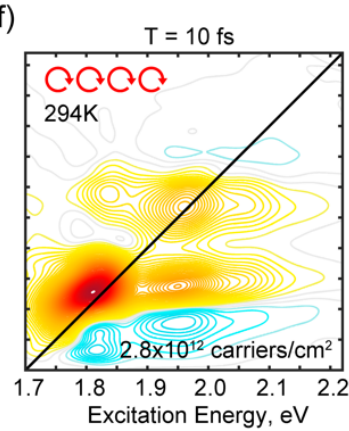

(c)

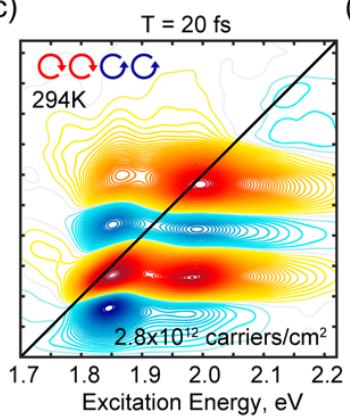

(g)

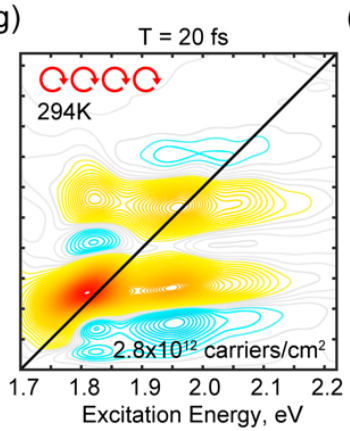

(d)

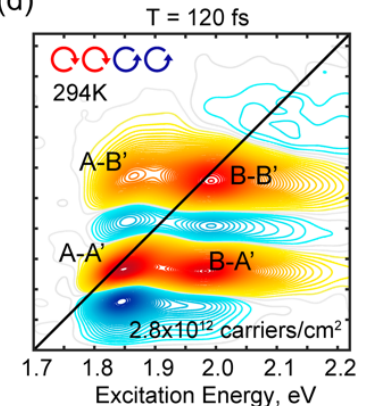

(h)

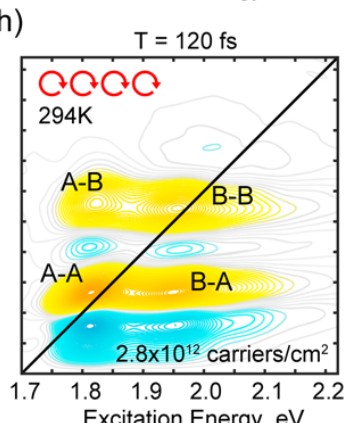

Figure S12. Absorptive 2DES maps for cross- (a-d) and co-polarized (e-h) room-temperature experiments at an estimated carrier density of $2.8 \times 10^{12}$ carriers $/ \mathrm{cm}^{2}$ for waiting time delays $\mathrm{T}=0,10,20$, and 120 fs. The spectra are normalized to the global maximum of the dataset for each experiment. The displayed data is an average of $n=4$ and $\mathrm{n}=6$ datasets for the cross- and co-polarized experiments, respectively.

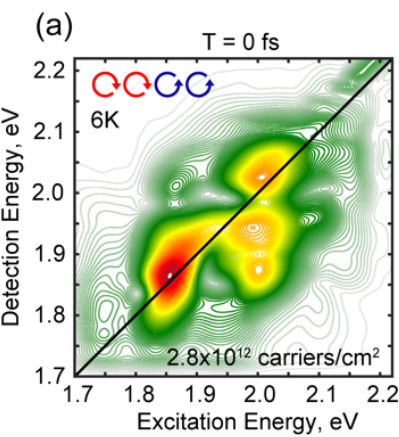

(e)

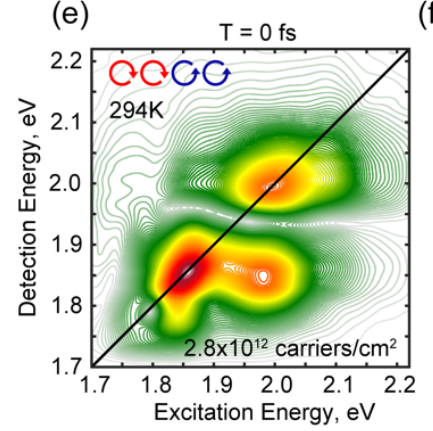

(b)

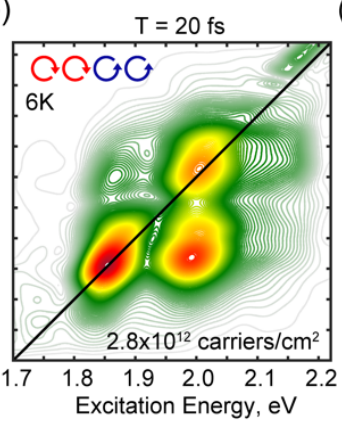

(f)

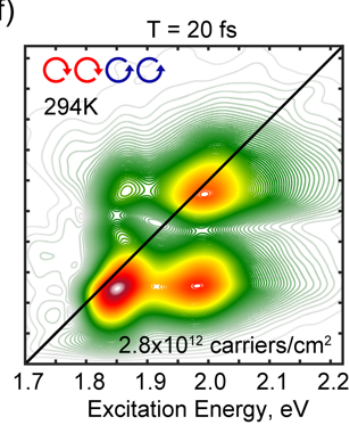

(c)

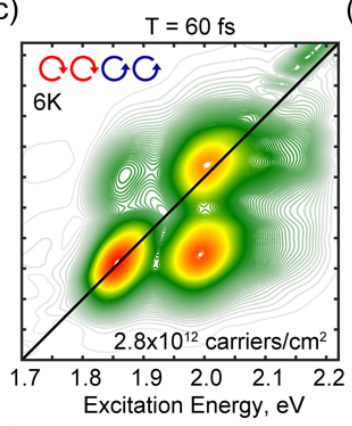

(g)

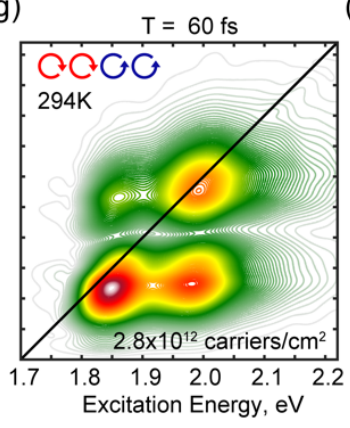

(d)

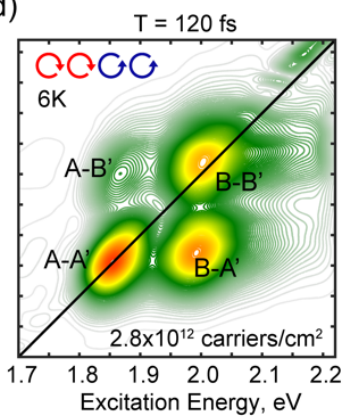

(h)

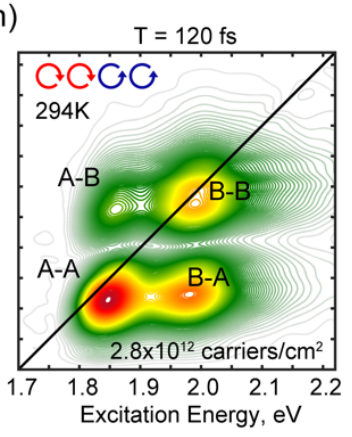

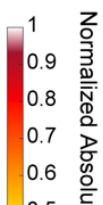

0.5 함

0.4 今

0.3 क

0.2 丞

0.1 할

Figure S13. Absolute value 2DES maps for cross-polarized experiments at 6K (a-d) and 294K (e-h) at an estimated carrier density of $2.8 \times 10^{12}$ carriers $/ \mathrm{cm}^{2}$ for waiting time delays $\mathrm{T}=0,20,60$, and $120 \mathrm{fs}$. Absolute value data are shown to facilitate a more direct comparison between the two experiments. The spectra are normalized to the global maximum of the dataset for each experiment. The displayed data is an average of $n=6$ and $n=4$ datasets for the cross-polarized experiments at cryogenic and room-temperature, respectively. 
(a)

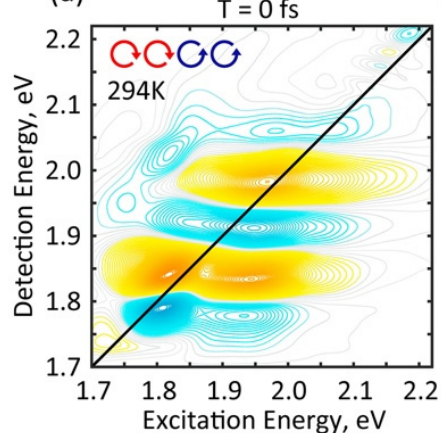

(d)

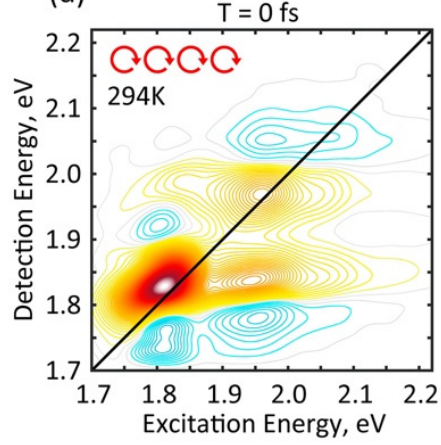

(b)

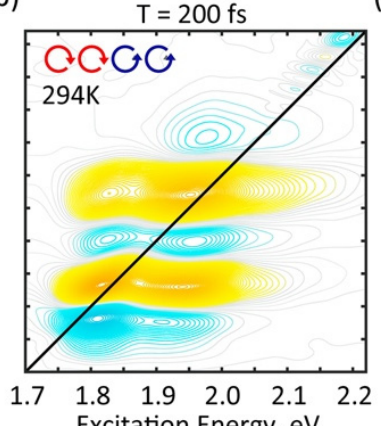

(e)

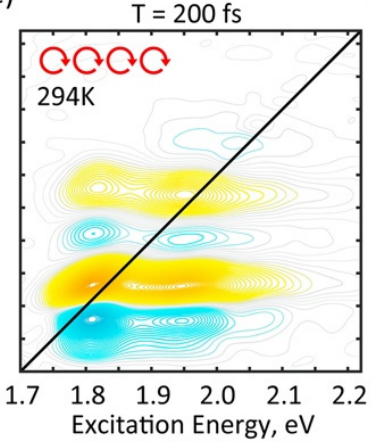

(c)

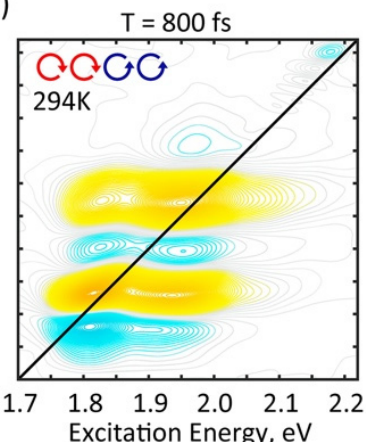

(f)

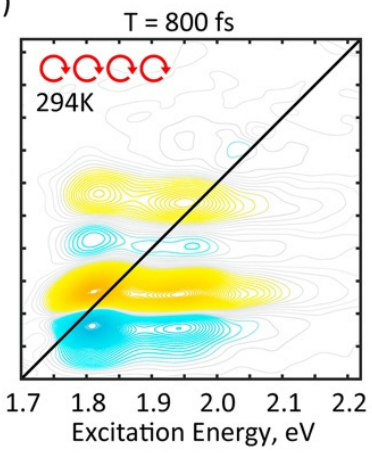

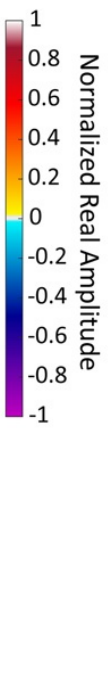

Figure S14. 2DES absorptive maps for cross- (a-c) and co- (d-f) circularly polarized pulse sequences at $294 \mathrm{~K}$ for waiting times $T=0,200$, and $800 \mathrm{fs}$. The spectra are normalized to $\mathrm{T}=800 \mathrm{fs}$ to highlight the similar relative intensities of the spectral features between the two polarization sequences after the initial dynamics in the first $100 \mathrm{fs}$. The displayed data is an average of $\mathrm{n}=8$, and $\mathrm{n}=3$ datasets for the cross- and co-polarized experiments, respectively. 


\section{Additional 2DES waiting time traces}
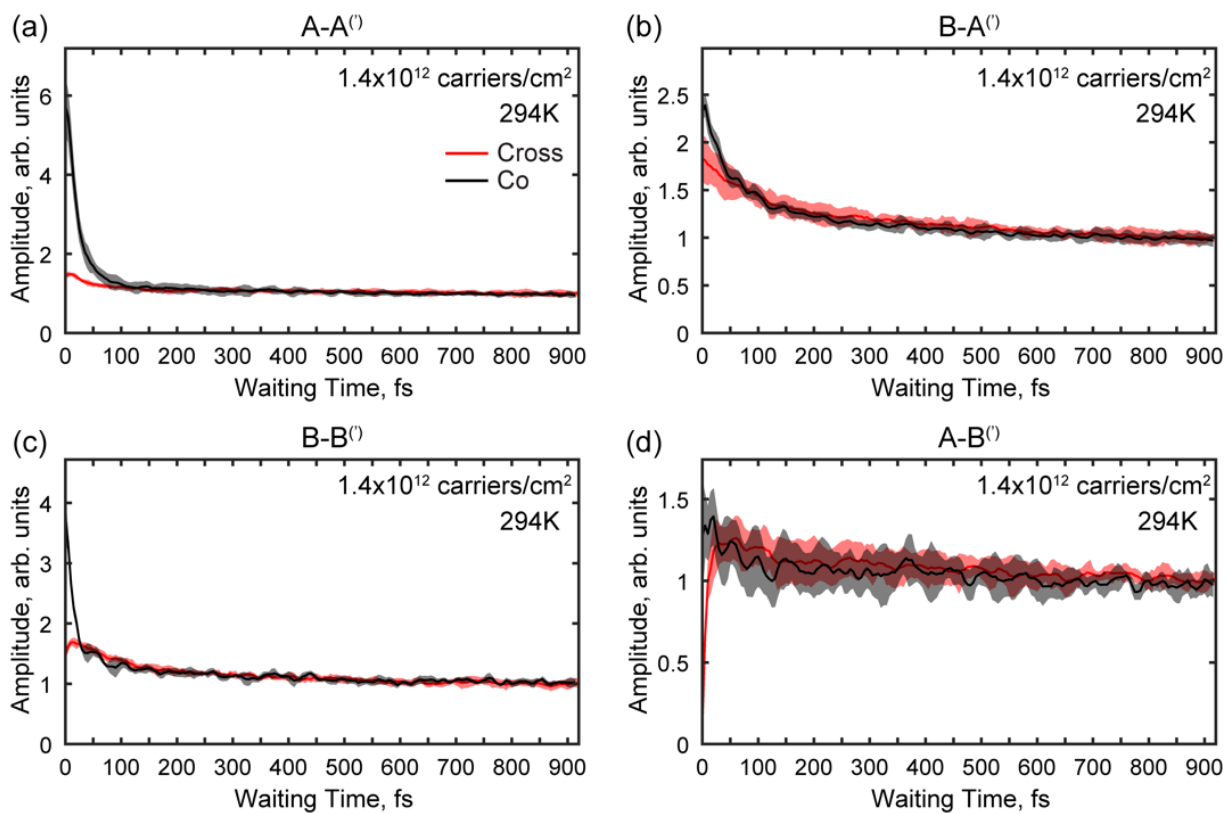

Figure S15. Representative data showing dynamics to $\sim 900$ fs for co- and cross-circularly polarized 2DES experiments at room-temperature. The data are normalized at 800 fs to highlight the nearly complete loss of the differences in the intra- versus intervalley dynamics by $\sim 100$ fs after optical excitation. The data show an estimated initial valley polarization of $\sim 60 \%$ (A exciton) and $\sim 40 \%$ (B exciton), defined as $P=\frac{\text { Co-Cross }}{\text { Co+Cross }}$. The lack of obvious subsequent signal growth in the cross-polarized waiting time traces indicates the initial mechanisms responsible for intervalley coupling likely occur during or immediately after optical excitation. The shaded regions correspond to the standard error of the mean.

(a)

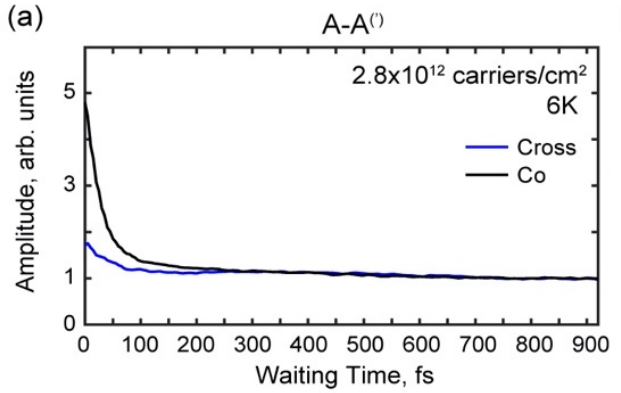

(c)

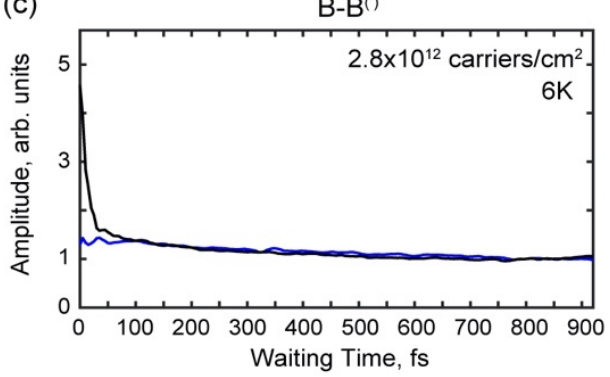

(b)

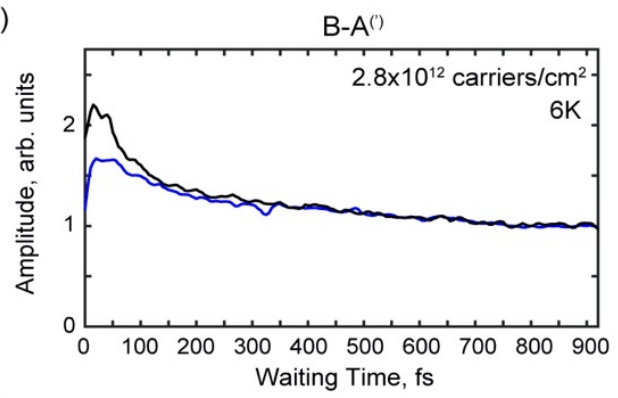

(d)

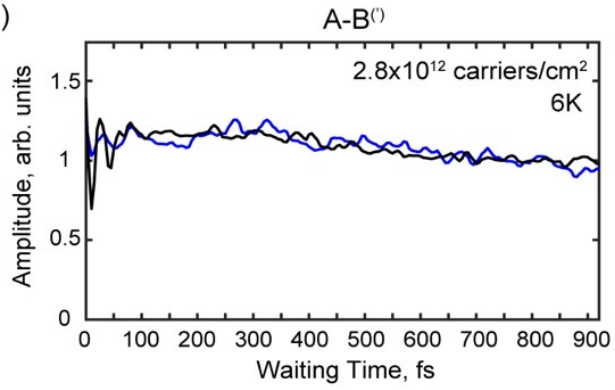

Figure S16. Representative data showing dynamics up to $\sim 900$ fs for co- and cross-circularly polarized experiments at cryogenic temperature normalized at $\mathrm{T}=800 \mathrm{fs}$. 
(a)

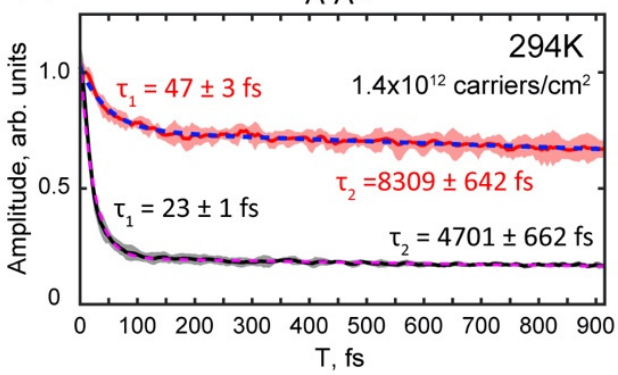

(c)

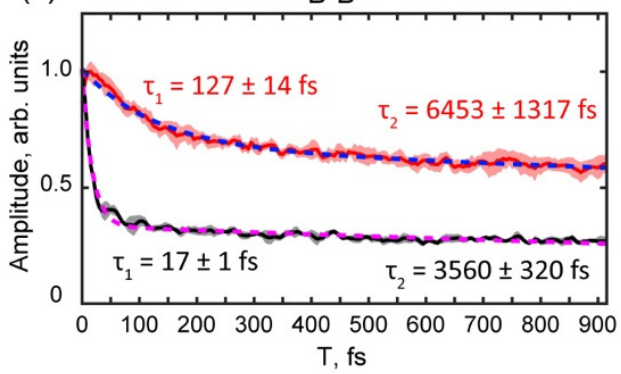

(b)

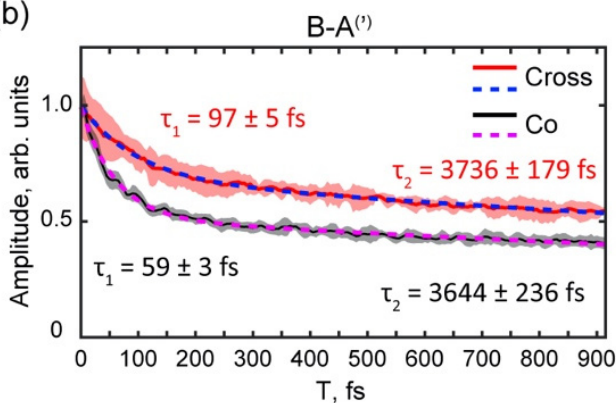

(d)

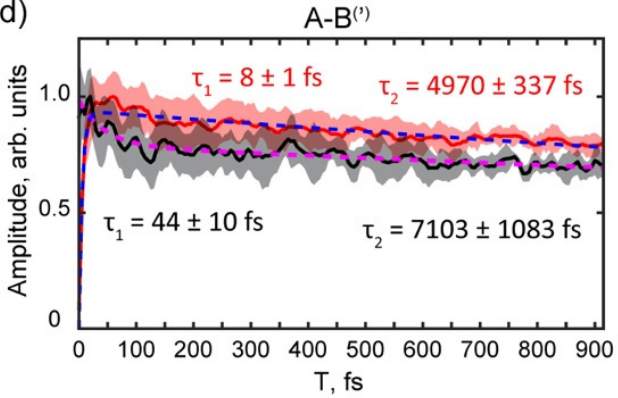

Figure S17. Kinetic fits to the time traces to $\sim 900 \mathrm{fs}$. Time constants from biexponential fits (dashed lines) to the data are shown. In panel (d) for the cross-polarized experiment, a single exponential rise and exponential decay is used as opposed to a biexponetial decay.

(a)

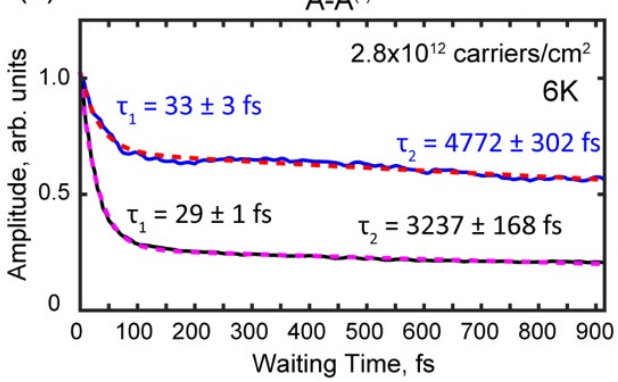

(c)

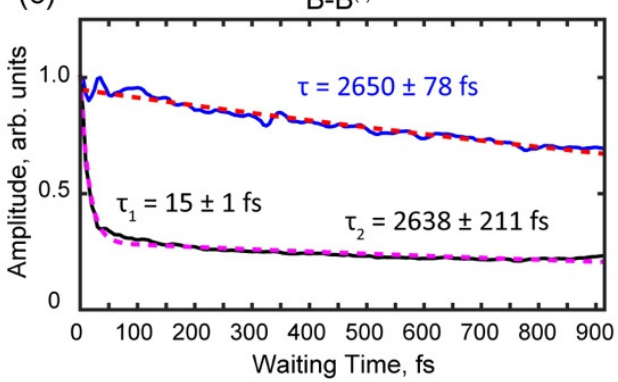

(b)

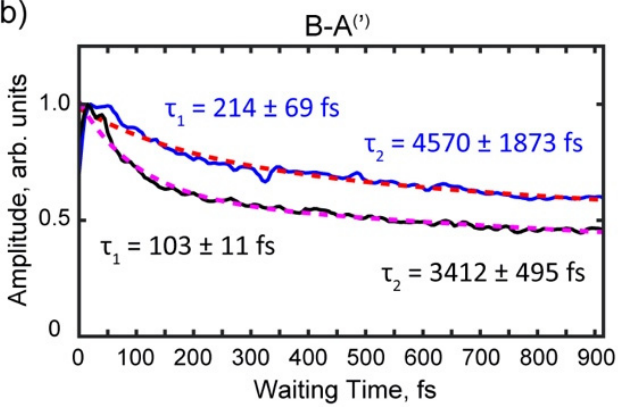

(d)

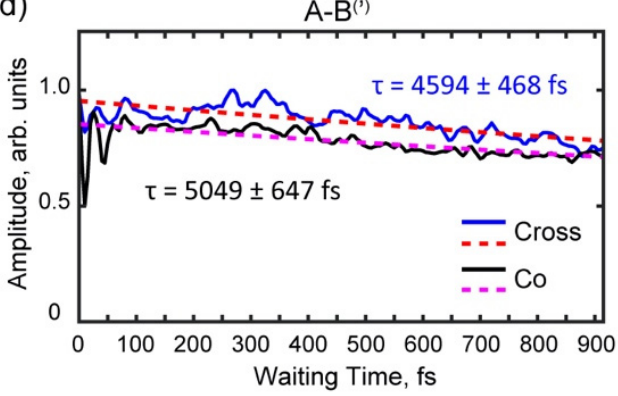

Figure S18. Kinetic fits to the cryogenic temperature time traces to $\sim 900 \mathrm{fs}$. Time constants from fits (dashed lines) to the data are shown. In panel (d) and in panel (c) for the cross-polarized experiment, a single exponential decay is sufficient to reproduce the dynamics. The biexponential decay in panel (b) does not capture the initial rise to peak amplitude within $\sim 20$ fs. 
(a)

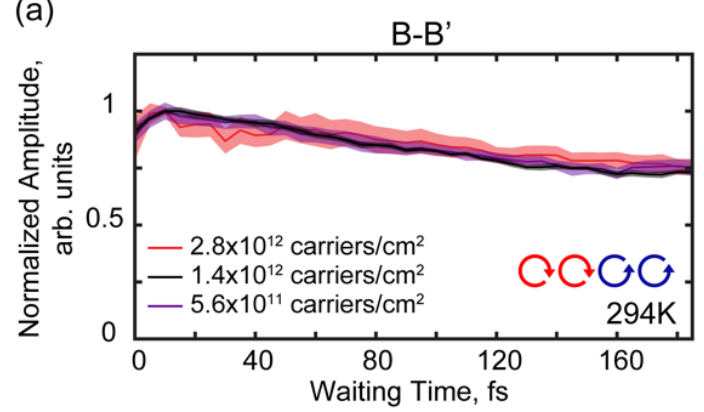

(c)

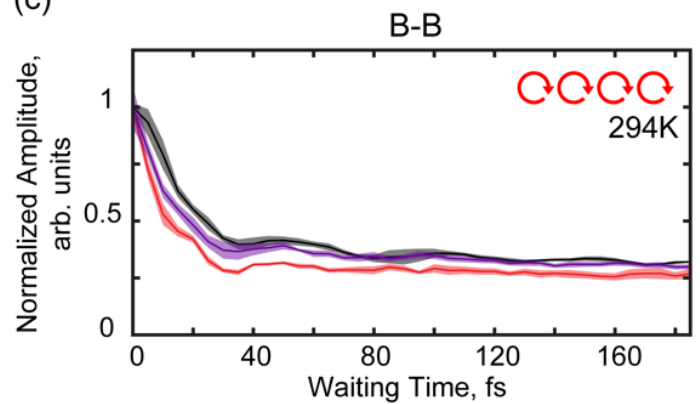

(b)

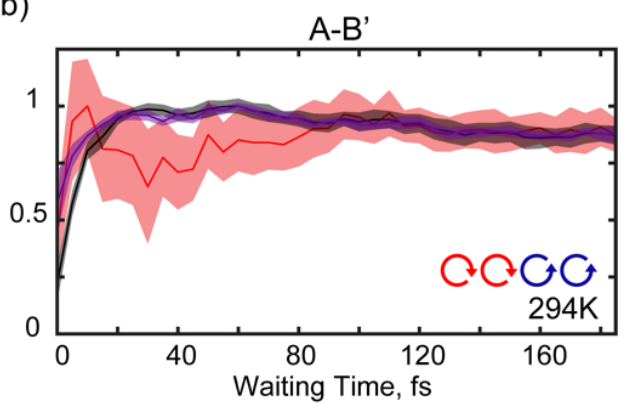

(d)

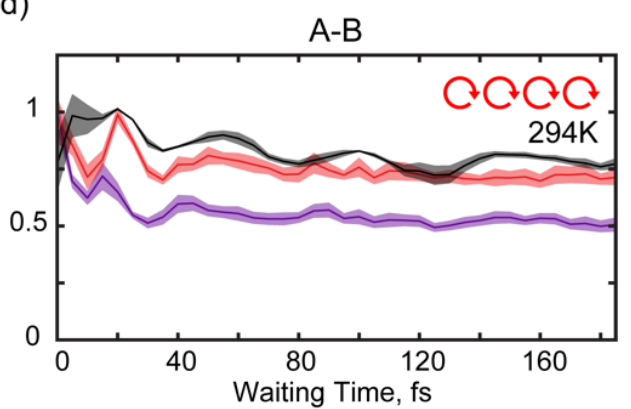

Figure S19. Waiting time traces for the B-B $\mathrm{B}^{\left({ }^{\circ}\right)}$ diagonal and A-B ${ }^{\left({ }^{\circ}\right.}$ upper cross-peak features for both cross- and cocircularly polarized pulse sequences at room-temperature. Multiple fluences are shown. Shaded error bars represent the standard error of the mean averaging $n$ datasets: $n=4, n=8$, and $n=6$ for cross-polarized datasets and $n=6, n=$ 3 , and $\mathrm{n}=5$ for co-polarized datasets for fluences of $2.8 \times 10^{12}, 1.4 \times 10^{12}$, and $5.6 \times 10^{11}$ carriers $/ \mathrm{cm}^{2}$, respectively.

(a)

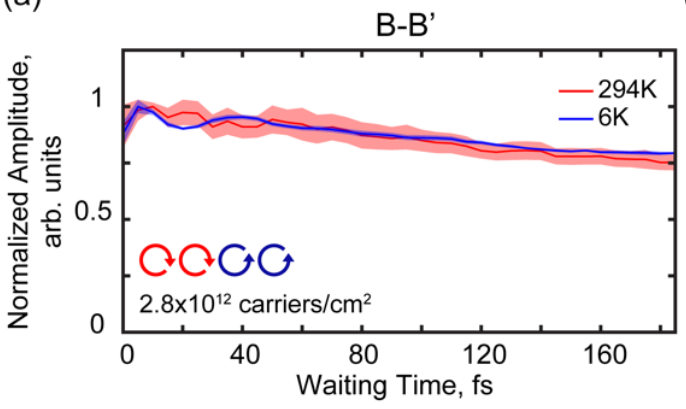

(c)

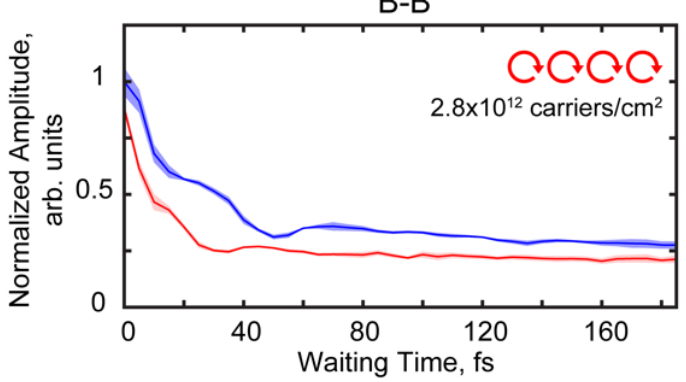

(b)

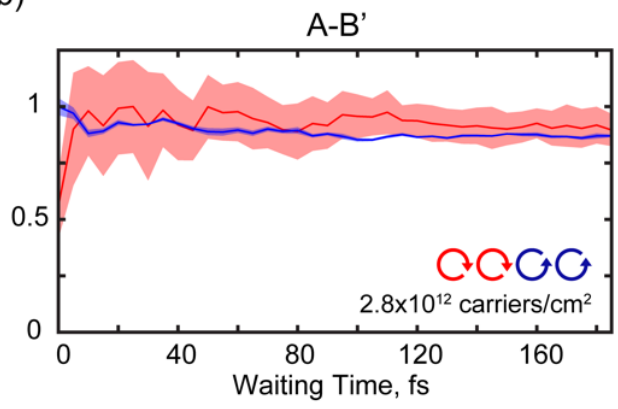

(d)

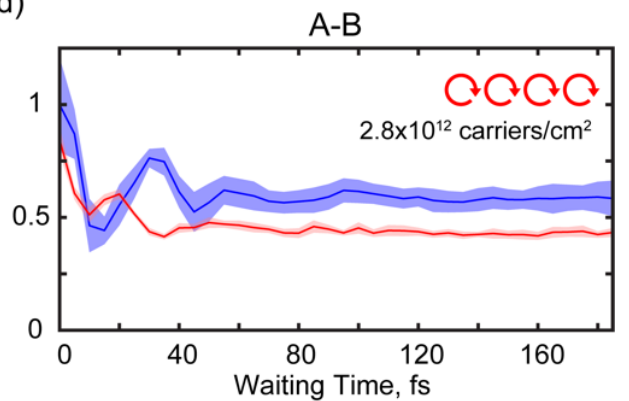

Figure S20. Waiting time traces for B-B $\mathrm{B}^{\left({ }^{\circ}\right.}$ diagonal and A-B $\mathrm{B}^{\left({ }^{\circ}\right.}$ upper cross-peak features for both cross- and cocircularly polarized pulse sequences at cryogenic temperature. Shaded error bars represent the standard error of the mean averaging $n$ datasets: $n=4$ and $n=6$ datasets for cross- and co-polarized pulse sequences, respectively. Absolutevalued data are shown. A single excitation fluence of $2.8 \times 10^{12}$ carriers $/ \mathrm{cm}^{2}$ was used in the cryogenic experiments. 

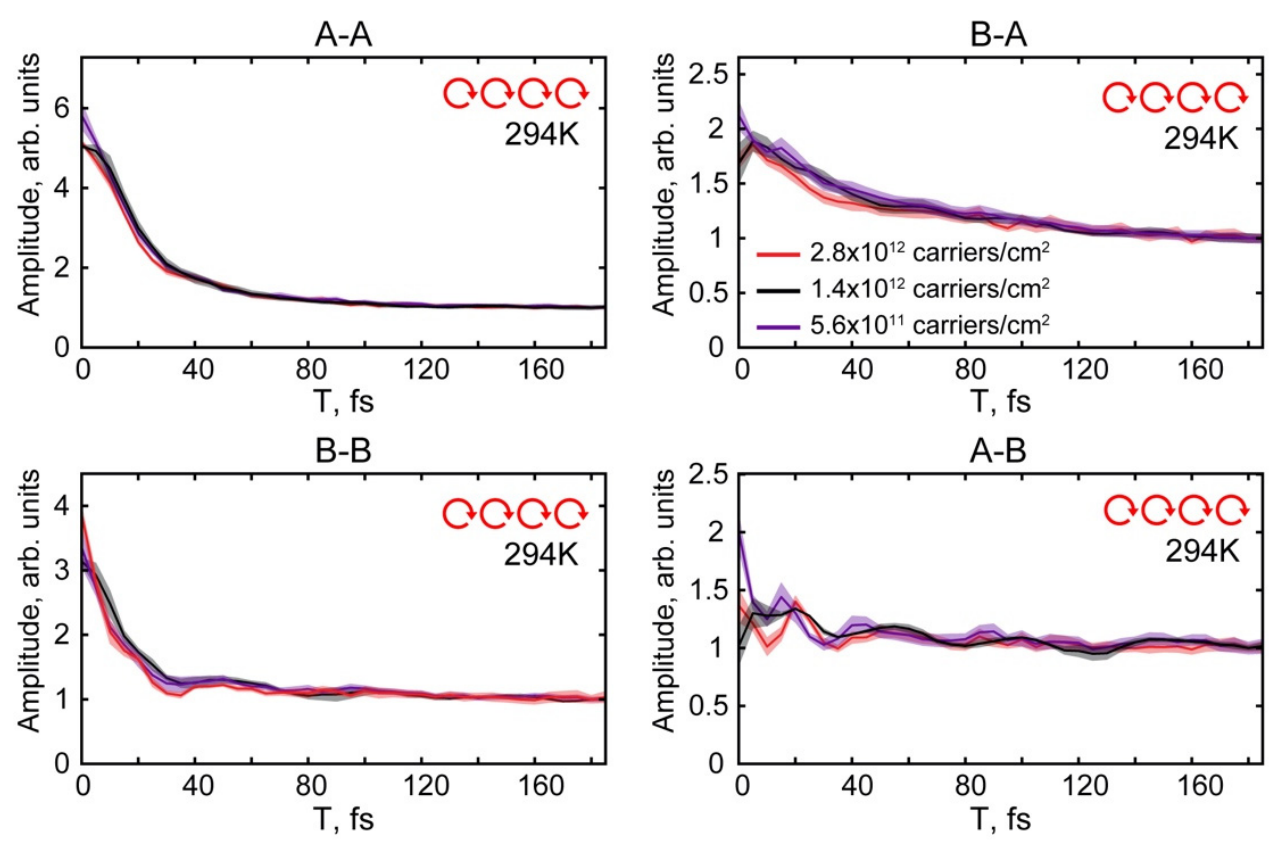

Figure S21. Waiting time traces normalized at $\mathrm{T}=180 \mathrm{fs}$ for the co-circularly polarized pulse sequences at roomtemperature. This presentation shows that the dynamics are largely within the error bars except for differences in intensity at $\mathrm{T} \sim 0 \mathrm{fs}$. Multiple excitation fluences are shown. Shaded error bars represent the standard error of the mean averaging $n$ datasets: $\mathrm{n}=6, \mathrm{n}=3$, and $\mathrm{n}=5$ for co-polarized datasets for fluences of $2.8 \times 10^{12}, 1.4 \times 10^{12}$, and $5.6 \times 10^{11}$ carriers $/ \mathrm{cm}^{2}$, respectively.
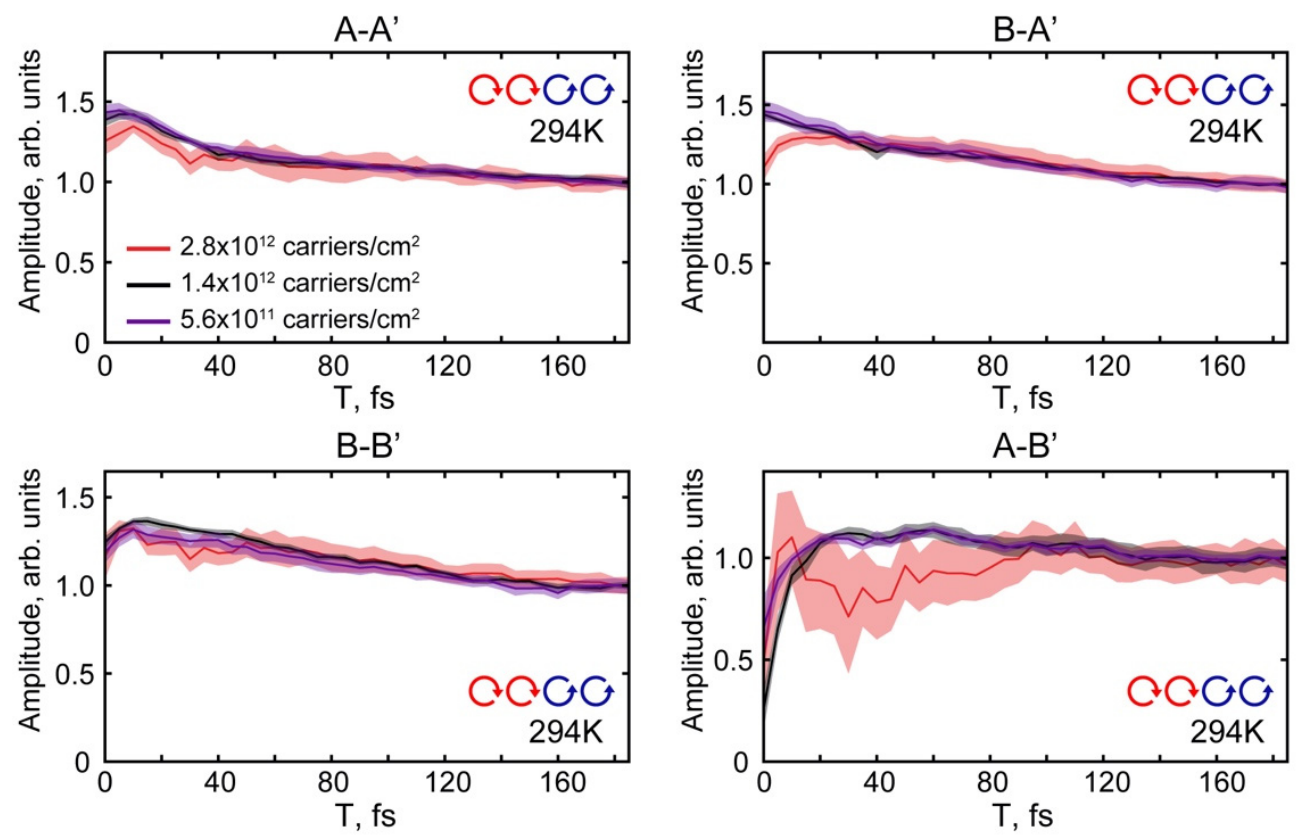

Figure S22. Waiting time traces normalized at $\mathrm{T}=180 \mathrm{fs}$ for the cross-circularly polarized pulse sequences at roomtemperature. This presentation shows that the dynamics are largely within the error bars. Multiple excitation fluences are shown. Shaded error bars represent the standard error of the mean averaging $n$ datasets: $n=4, n=8$, and $n=6$ for cross-polarized datasets for fluences of $2.8 \times 10^{12}, 1.4 \times 10^{12}$, and $5.6 \times 10^{11}$ carriers $/ \mathrm{cm}^{2}$, respectively. 
We present representative 2DES waiting time traces of the cross-polarized experiment showing dynamics for $\mathrm{T}<0$ fs in Figure S24. The data display finite signal at $\mathrm{T}<0$ fs and a rise time outside of the instrument response function (IRF). A fit to the data (eqn. 1) convolved with the $\mathrm{IRF}^{1}$ does not reproduce the dynamics well, indicating the signal amplitude at negative delay times does not fully arise from the finite IRF. Pulse-misordering artifacts when scanning the first time delay $\tau$ at $\mathrm{T}<0$ fs will give rise to finite signal at negative delay times. This signal at $\mathrm{T}<0$ fs is absent in the transient grating trace in Figure S23 where $\tau=0$ fs. Despite this noninstantaneous rise, the intervalley coupling signals do not exhibit any further growth dynamics after $\mathrm{T} \sim 10 \mathrm{fs}$, providing evidence for intervalley coupling on the timescale of optical excitation.

$$
\left.S \propto\left(1-e^{-\frac{T}{\tau_{r i s e}}}\right) \times \text { Heaviside }(T=0) \times\left(A_{1} e^{-\frac{T}{\tau_{1}}}+A_{2} e^{-\frac{T}{\tau_{2}}}\right)\right) \quad \text { (eqn. 1) }
$$
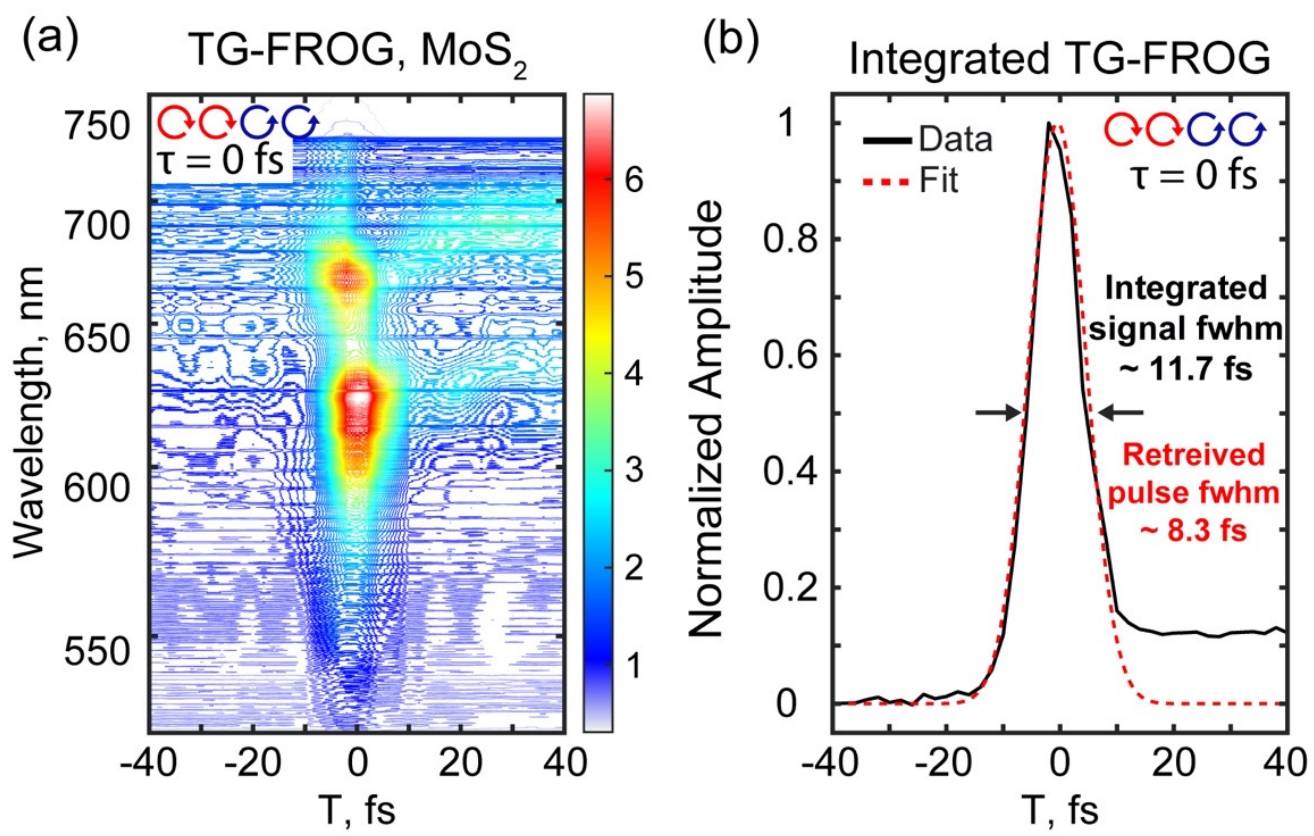

Figure S23. TG-FROG of $\mathrm{MoS}_{2}$ taken directly before 2DES measurements. The waiting time $\mathrm{T}$ is scanned while $\tau=$ $0 \mathrm{fs}$. A clear IRF is resolved from the non-resonant response at $\mathrm{T} \sim 0 \mathrm{fs}$ with clear signal amplitude present for $\mathrm{T}>0$ fs. In contrast to the 2DES time traces (Figure S24), there is negligible signal amplitude outside of the IRF at T $<0$ fs. 
(a)

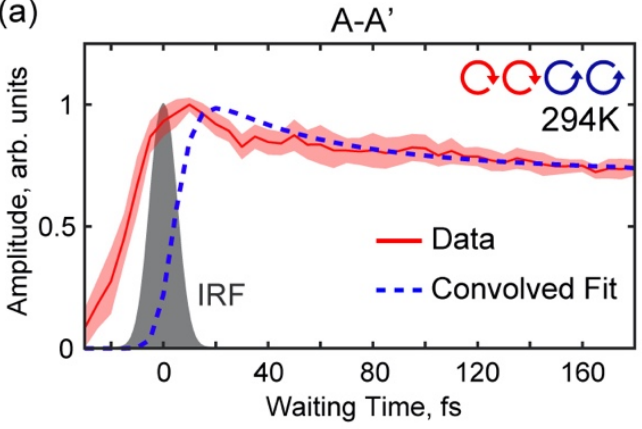

(c)

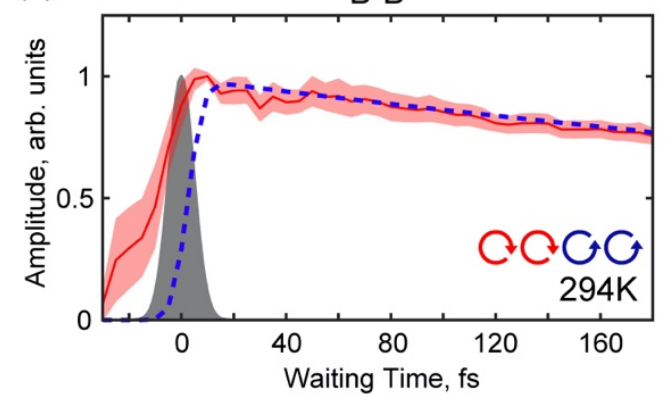

(b)

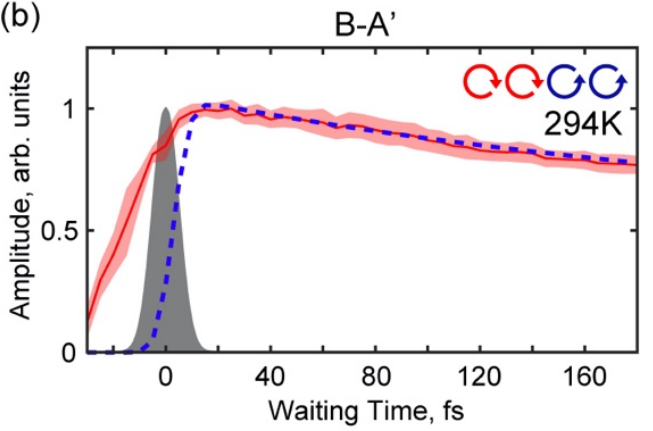

(d)

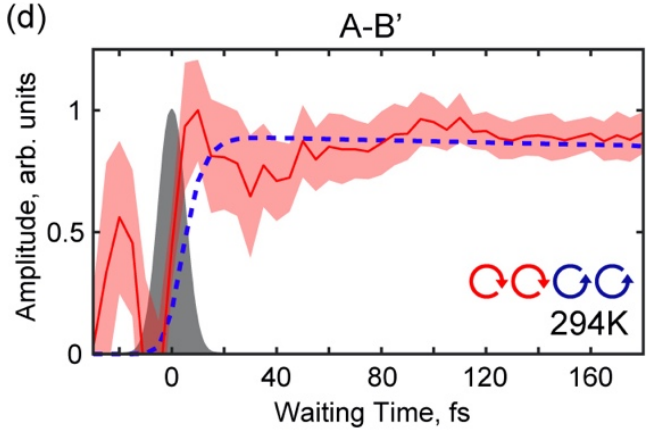

Figure S24. Waiting Time traces for the cross-polarized experiment including $\mathrm{T}<0$ fs with an overlaid estimated IRF (12 fs fwhm, See Figures S5, S6, S23) and a fit to the data convolved with the IRF (eqn. 1).

\section{2DES measurements on strain-relieved sample growths}

To probe whether the observed spectral features are consistent between sample growths or dependent on factors such as grain size and intrinsic strain, we performed 2DES measurements at room-temperature on two additional samples with different grain sizes ( $\sim 2 \mu \mathrm{m}$ and $\sim 0.2 \mu \mathrm{m})$ than the sample in the main text $(\sim 1 \mu \mathrm{m})$. Additionally, these two samples were stamp-transferred from the growth substrate to relieve the intrinsic strain acquired during the growth process (see Figure S1). Figures S25, S26 show qualitatively similar spectral features at short time delay in both the co- and cross-polarized pulse sequences to the data in the main text (Figures 2, 4). The crosspolarized spectra show strong signal at short time delays that slowly decays, with the A-A' diagonal and B-A' lower cross-peak being similar in relative intensity. Conversely, the copolarized spectra display a prominent A-A diagonal feature that rapidly decays within tens of femtoseconds. Based on these additional experiments on samples of differing grain size, defect density, and strain, the observed ultrafast intervalley coupling is likely an inherent feature of largearea MOCVD-grown systems. Further theoretical and experimental work may be required to determine the degree to which the observed intervalley coupling differs in exfoliated or 'pristine' samples with low defect density, as well as spatially across sample domains. 
Sample 2 : $\sim 2 \mu \mathrm{m}$ grain size

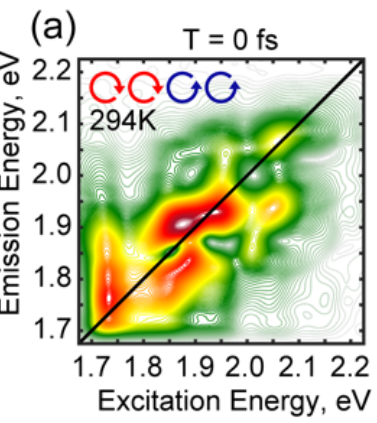

(b)

(c)

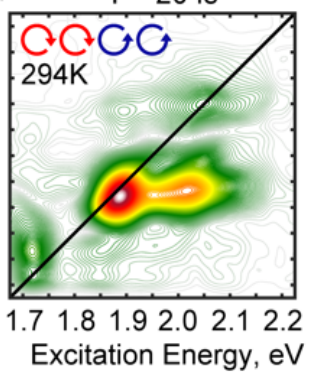

(d)

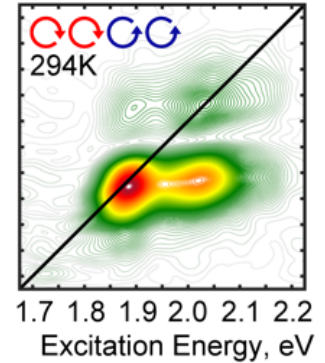

(e)

(f)

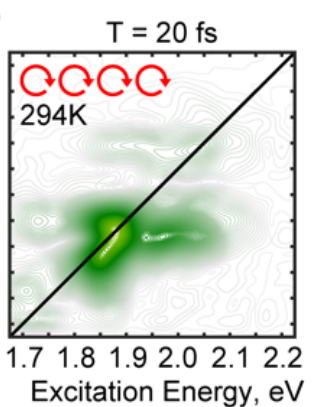

(g)

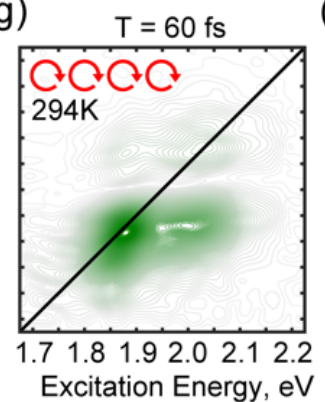

(d) $\quad T=120 \mathrm{fs}$

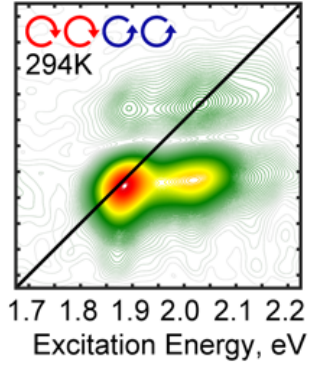

(h)

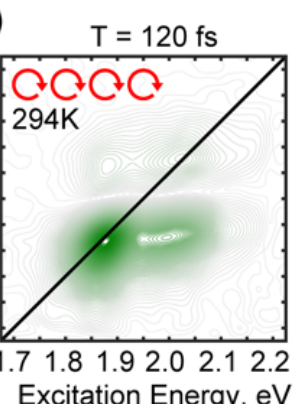

Figure S25. Cross- and co-polarized 2DES measurements of a stamp-transferred MOCVD-grown sample with $\sim 2 \mu \mathrm{m}$ grain size. The spectra are normalized to the maximum of the respective dataset.

\section{Sample $3: \sim 0.2 \mu \mathrm{m}$ grain size}

(a)

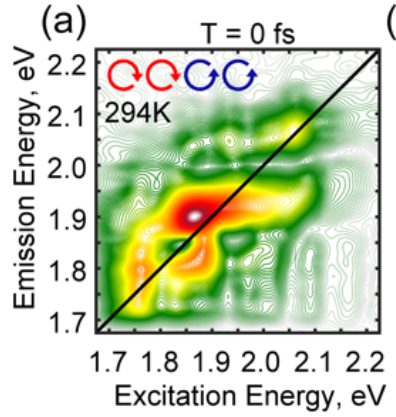

(e)

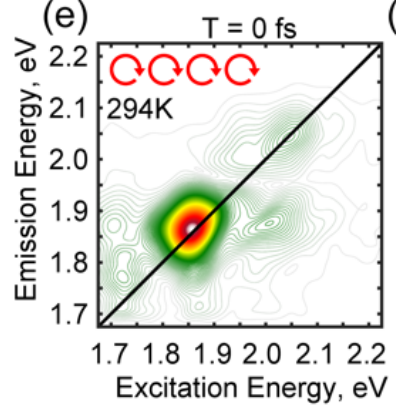

(b)
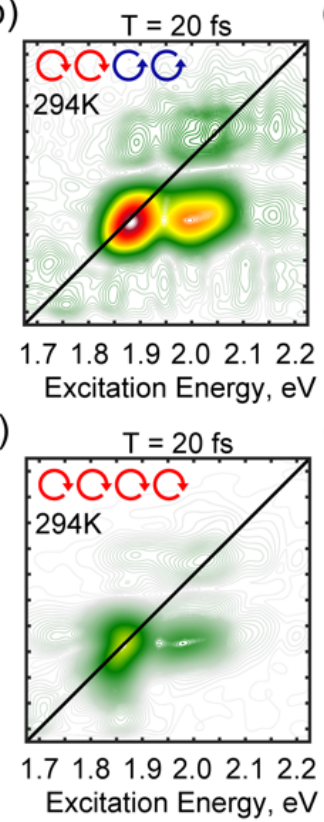

(c)

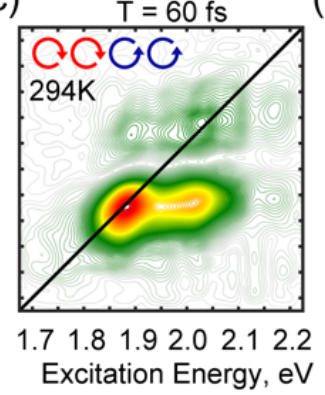

(g)

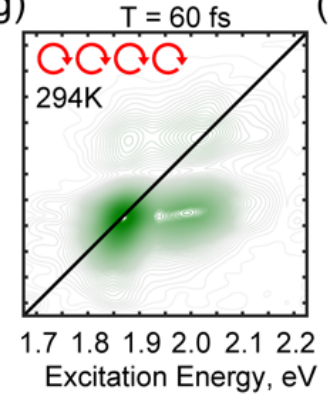

(d)

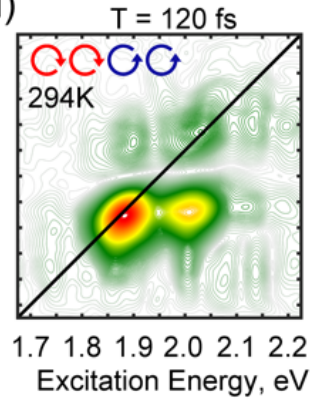

(h)

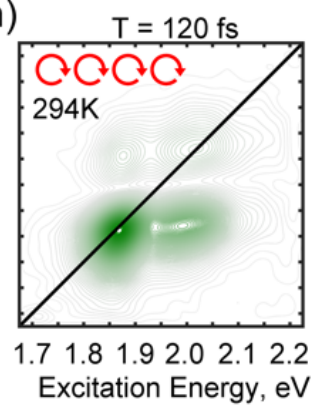

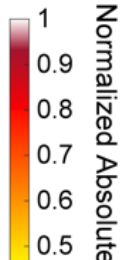

$0.4 \underset{3}{\widehat{S}}$

$0.3 \stackrel{0}{\frac{2}{5}}$

0.2 买

0.1 흘

Figure S26. Cross- and co-polarized 2DES measurements of a stamp-transferred MOCVD-grown sample with $\sim 0.2 \mu \mathrm{m}$ grain size. The spectra are normalized to the maximum of the respective dataset. 


\section{Estimating the carrier-density in 2DES measurements}

To estimate the excited carrier density in the 2DES experiments, we follow the procedure of Guo et al. ${ }^{2}$ The linear absorption spectrum is converted to percent absorbance and the laser spectrum to number of photons at a given wavelength using the measured pulse energy (Figure S27). Pulse energies of $7 \mathrm{~nJ}, 3.5 \mathrm{~nJ}$, and $1.5 \mathrm{~nJ}$ were used in the room temperature 2DES experiments, and $7 \mathrm{~nJ}$ pulse energies were used at cryogenic temperature. Multiplying these spectra together, integrating over the wavelength dimension, and dividing by the spot size $(\sim 290 \mu \mathrm{m})$ gives the estimated total number of carriers per $\mathrm{cm}^{2}$ generated in the 2DES experiments.

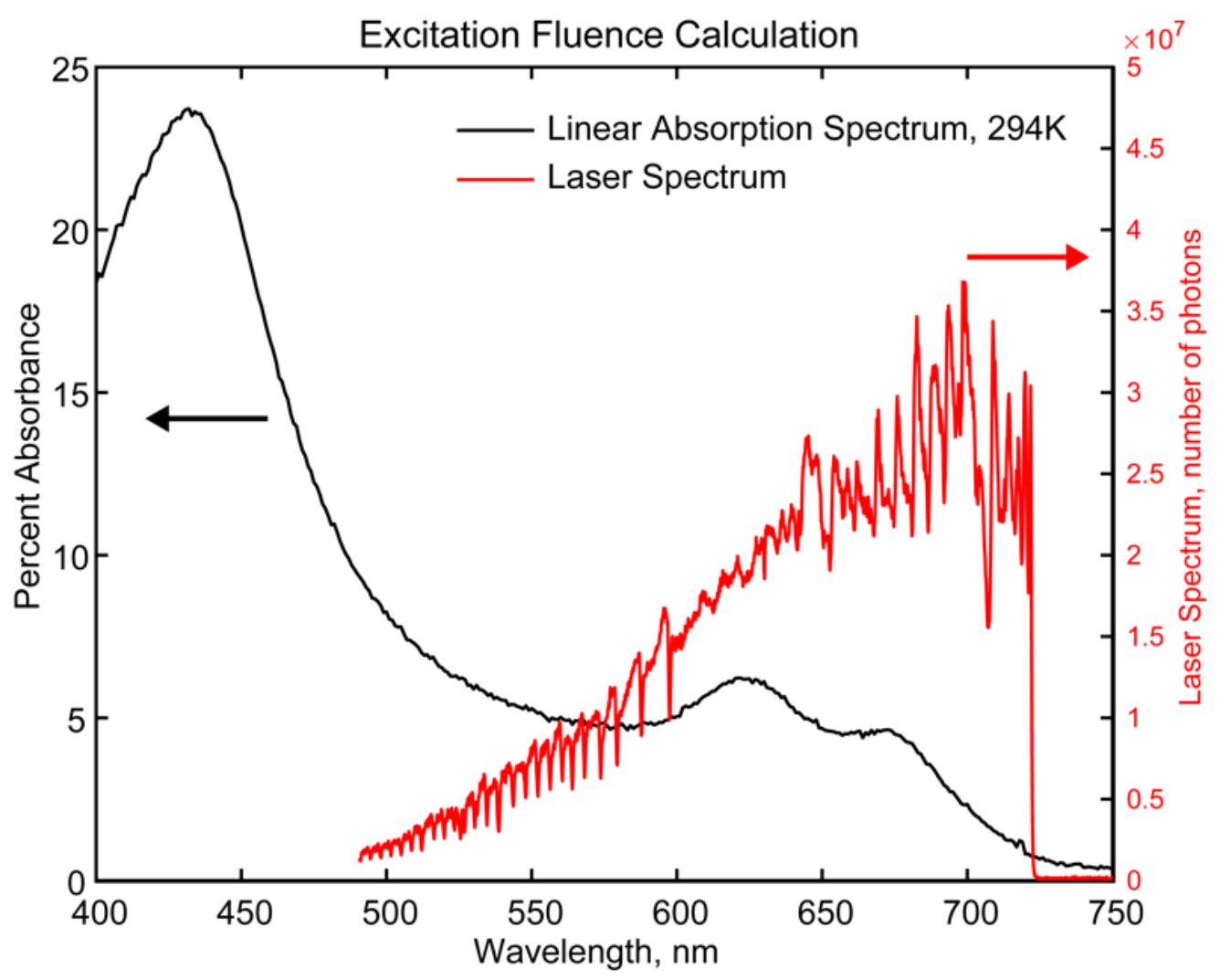

Figure S27. Linear absorption spectrum of the monolayer $\mathrm{MoS}_{2}$ sample expressed as percent absorbance and the laser spectrum used in experiments, converted to number of based on the pulse energy ( $3.5 \mathrm{~nJ} /$ pulse shown here). Integrating over the product of the two spectra is used to estimate the total carrier density generated during experiments. The room-temperature absorption spectrum was also used to calculate excitation densities for the cryogenic experiments. 


\section{Coupling features and cross-peaks in two-dimensional spectroscopy}

In two-dimensional spectroscopy, cross-peaks are indicative of energy transfer between states or coupling between distinct transitions, such as sharing a common ground state with transition dipole strength into both excited states. The latter is especially identified as the presence of both upper and lower off-diagonal cross-peaks at $\mathrm{T}=0 \mathrm{fs}$ in $2 \mathrm{D}$ spectra before dynamic processes such as energy transfer occur. In this case, excitation of one state results in bleaching of the other state due to their shared level. It is important to note that the spectral features observed in experiments are the resulting interference between many possible Feynman pathways that describe the material response. In material systems, for example, excitation-induced effects may cause peak broadening or energy level shifts as a function of excitation density, resulting in nontrivial dynamic interference of multiple pathways that lead to the appearance of off-diagonal features. However, Guo et al. ${ }^{2}$ attribute upper and lower intravalley cross-peaks in co-polarized 2DES spectra of $\mathrm{MoS}_{2}$ to a time-dependent exchange-mediated mixing of the A and $\mathrm{B}$ exciton levels but rule out excitation-induced dephasing as the origin of these features. In general, however, excitation-induced effects have been shown to be strong in TMD systems, including bandgap renormalization and excitation-induced dephasing. ${ }^{3-7}$ The former leads to a timedependent shift of the transition energy manifesting as a red-shifted photo-induced absorption feature while the latter gives rise to broadened spectral features.

In the cross-polarized 2D spectra presented in this work, features arising both on and off the main diagonal require coupling between the $\mathrm{K}$ and $\mathrm{K}$ ' valleys but do not necessarily require carrier or population transfer. Importantly, the transitions $\mathrm{K}$ and $\mathrm{K}$ ' are distinct in momentum space and predicted to be uncoupled transitions, so excitation of one valley is not expected to result in a bleach of the other. In particular, the appearance of these features at short time delay ( $\mathrm{T} \sim 0 \mathrm{fs}$ ) is significantly faster than the multitude of dynamic processes described previously. Higher order (multi-) exciton effects and renormalization effects also occur at short time delay on the timescale of excitation as described in the main text.

\section{References}

1. Trovatello, C.; Katsch, F.; Borys, N. J.; Selig, M.; Yao, K.; Borrego-Varillas, R.; Scotognella, F.; Kriegel, I.; Yan, A.; Zettl, A.; Schuck, P. J.; Knorr, A.; Cerullo, G.; Conte, S. D., The Ultrafast Onset of Exciton Formation in 2D Semiconductors. Nat. Commun. 2020, 11, 5277.

2. Guo, L.; Wu, M.; Cao, T.; Monahan, D. M.; Lee, Y.-H.; Louie, S. G.; Fleming, G. R., Exchange-Driven Intravalley Mixing of Excitons in Monolayer Transition Metal Dichalcogenides. Nat. Phys. 2018, 15, 228-232.

3. Katsch, F.; Selig, M.; Knorr, A., Exciton-Scattering-Induced Dephasing in TwoDimensional Semiconductors. Phys. Rev. Lett. 2020, 124, 257402.

4. Smejkal, V.; Libisch, F.; Molina-Sanchez, A.; Trovatello, C.; Wirtz, L.; Marini, A., TimeDependent Screening Explains the Ultrafast Excitonic Signal Rise in 2D Semiconductors. ACS Nano 2021, 15, 1179-1185. 
5. Pogna, E. A.; Marsili, M.; De Fazio, D.; Dal Conte, S.; Manzoni, C.; Sangalli, D.; Yoon, D.; Lombardo, A.; Ferrari, A. C.; Marini, A.; Cerullo, G.; Prezzi, D., Photo-Induced Bandgap Renormalization Governs the Ultrafast Response of Single-Layer $\mathrm{MoS}_{2}$. ACS Nano 2016, 10, 1182-1188.

6. Cunningham, P. D.; Hanbicki, A. T.; McCreary, K. M.; Jonker, B. T., Photoinduced Bandgap Renormalization and Exciton Binding Energy Reduction in $\mathrm{WS}_{2}$. ACS Nano 2017, 11, 12601-12608.

7. $\quad$ Moody, G.; Kavir Dass, C.; Hao, K.; Chen, C. H.; Li, L. J.; Singh, A.; Tran, K.; Clark, G.; $\mathrm{Xu}, \mathrm{X}$; Berghauser, G.; Malic, E.; Knorr, A.; Li, X., Intrinsic Homogeneous Linewidth and Broadening Mechanisms of Excitons in Monolayer Transition Metal Dichalcogenides. Nat. Commun. 2015, 6, 8315. 